\title{
Linear Satisfiability Preserving Assignments
}

\author{
Kei Kimura \\ Toyohashi University of Technology \\ Toyohashi, 441-8580, Japan \\ Kazuhisa Makino \\ Kyoto University \\ Kyoto, 606-8502, Japan
}

KIMURA@CS.TUT.AC.JP

MAKINO@KURIMS.KYOTO-U.AC.JP

\begin{abstract}
In this paper, we study several classes of satisfiability preserving assignments to the constraint satisfaction problem (CSP). In particular, we consider fixable, autark and satisfying assignments. Since it is in general NP-hard to find a nontrivial (i.e., nonempty) satisfiability preserving assignment, we introduce linear satisfiability preserving assignments, which are defined by polyhedral cones in an associated vector space. The vector space is obtained by the identification, introduced by Kullmann, of assignments with real vectors. We consider arbitrary polyhedral cones, where only restricted classes of cones for autark assignments are considered in the literature. We reveal that cones in certain classes are maximal as a convex subset of the set of the associated vectors, which can be regarded as extensions of Kullmann's results for autark assignments of CNFs. As algorithmic results, we present a pseudo-polynomial time algorithm that computes a linear fixable assignment for a given integer linear system, which implies the well known pseudo-polynomial solvability for integer linear systems such as two-variable-per-inequality (TVPI), Horn and q-Horn systems.
\end{abstract}

\section{Introduction}

We in this section introduce central concepts in the study and summarize our results.

\subsection{Constraint Satisfaction Problem}

The constraint satisfaction problem (CSP, in short) is given as a triple $(V, D, \mathcal{C})$, where $V$ is a set of variables, $D$ is a finite variable domain, and $\mathcal{C}$ is a set of constraints on the variables that specify the permitted combinations of value assignments to variables. A solution to a CSP instance is an assignment to all the variables that satisfies all the constraints. The CSP is the problem of finding a solution to a given instance $(V, D, \mathcal{C})$. The CSP can express a number of problems in diverse fields, and is recognized as one of the most fundamental problems in computer science (e.g., Tsang, 1993; Creignou, Khanna, \& Sudan, 2001; Dechter, 2003; Rossi, van Beek, \& Walsh, 2006). The CSP has been studied extensively from both theoretical and practical points of view. For example, it is known that the CSP of a fixed finite constraint language has a dichotomy property (Schaefer, 1978; Bulatov, 2006, 2017; Zhuk, 2017). Namely, it is polynomially solvable if all the constraints satisfy a certain property, or otherwise NP-hard. Moreover, due to practical importance, many heuristic algorithms have been proposed (Tsang, 1993; Dechter, 2003; Rossi et al., 2006). In this paper, each constraint might involve a non-fixed number of variables, as is often assumed in the literature (van Hoeve \& Katriel, 2006). 
The Boolean satisfiability problem (SAT) can be regarded as a Boolean CSP, in which each constraint is represented by a clause (i.e., disjunction of literals). SAT itself is an important problem in computer science. It is well-known that SAT is NP-hard (Cook, 1971), but several restricted classes such as 2-SAT (Even, Itai, \& Shamir, 1976), Horn SAT (Henschen \& Wos, 1974), renamable Horn SAT (Lewis, 1978), q-Horn SAT (Boros, Crama, \& Hammer, 1990), and LinAut SAT (Kullmann, 2000; van Maaren, 2000) are solvable in polynomial time.

Integer linear systems are also formulated as a CSP. The problem is, given a matrix $G \in \mathbb{Z}^{m \times n}$, a vector $h \in \mathbb{Z}^{m}$, and a positive integer $d$, where $\mathbb{Z}$ denotes the set of all integers, to compute an integer vector $x \in\{0,1, \ldots, d\}^{n}$ such that $G x \geq h$. It is a well-studied topic, especially in the field of mathematical programming. The problem is strongly NP-hard, but several (semi-)tractable subclasses are known to exist. For example, the problem can be solved in polynomial time, if $n$ is bounded by some constant (Lenstra, Jr., 1983), or if $G$ is totally unimodular (Hoffman \& Kruskal, 1956). Moreover, it can be solved in pseudo-polynomial time if (1) $m$ is bounded by some constant (Papadimitriou, 1981), (2) $G$ corresponds to a two-variable-per-inequality (TVPI) system (i.e., each row of $G$ contains at most two nonzero elements) (Hochbaum, Megiddo, Naor, \& Tamir, 1993; Bar-Yehuda \& Rawitz, 2001), (3) $G$ is Horn (i.e., each row of $G$ contains at most one positive element) (Glover, 1964; van Maaren \& Dang, 2002), or (4) $G$ is q-Horn (Kimura \& Makino, 2016). It is also known that the problem is weakly NP-hard, even if $m$ is bounded by some constant or the system is TVPI and Horn (also called monotone quadratic) (Lagarias, 1985).

\subsection{Satisfiability Preserving Assignments}

As mentioned above, many practical algorithms have been proposed to solve CSPs (including SAT and integer linear systems). One of the important methods is based on reducing the problem size by partial variable assignments. For example, satisfiability preserving assignments are used to reduce the problem size, where a partial assignment is called satisfiability preserving if the satisfiability of the problem does not change after substituting it to the corresponding variables. Once a satisfiability preserving assignment is available, instead of solving the original problem instance, we may solve the problem instance obtained from the original one by the partial assignment. Here we note that the size of the resulting problem instance is smaller than the original one.

Satisfiability preserving assignments have been considered in many fields such as artificial intelligence and optimization. An important example is forced assignments, which are essentially the same concepts as back bones (Monasson, Zecchina, Kirkpatrick, Selman, \& Troyansky, 1999), frozen variables (Jonsson \& Krokhin, 2004), and implied values (Bordeaux, Cadoli, \& Mancini, 2008). An assignment of $a \in D$ to variable $x$ is called forced if $x$ has to be set to $a$ in all satisfying assignments. Unit clause propagation in SAT is a prominent example of forced assignments. Pure literals in SAT can also be seen as another example of satisfiability preserving assignments, where a literal $\ell$ is called pure if a given $\mathrm{CNF}$ contains no $\bar{\ell}$ (i.e., negation of $\ell$ ). By definition, for a pure literal $\ell$, an assignment $\ell=1$ is satisfiability preserving. Pure literals, together with unit clause propagation, are used by the Davis-Putnam-Logemann-Loveland (DPLL) algorithm (Davis \& Putnam, 1960; Davis, Logemann, \& Loveland, 1962) to reduce the search space. We note that the 
DPLL algorithm is a basis of many current SAT solvers. Also in practice, various types of satisfiability preserving assignments are used, especially in branch-and-bound algorithms. Among them, we consider three fundamental satisfiability preserving assignments, namely, satisfying, fixable, and autark partial assignments. A satisfying partial assignment is a straightforward example of satisfiability preserving assignments. A partial assignment is satisfying if it satisfies all the constraints no matter what values are assigned to the unassigned variables. Clearly, satisfying partial assignments are satisfiability preserving. An assignment $A$ to variable set $X \subseteq V$ is called fixable (Bordeaux et al., 2008; also called safe by, e.g., Kleine Büning \& Kullmann, 2009) if every satisfying total assignment stays satisfying, even if the assignment to $X$ is replaced by $A$, where the assignment to $V \backslash X$ remains unchanged. By definition, a fixable assignment is satisfiability preserving. Another example is autark assignments, which play an important role in this paper and thus will be stated in depth in the next paragraph.

For the SAT problem, autark assignments were introduced by Monien and Speckenmeyer (1985) to provide a fast exponential time algorithm. A partial assignment is called autark if it satisfies all the clauses that contain variables assigned by it. Later, Kullmann (2000) investigated autark assignments in terms of resolution refutation. A CNF is lean if it does not have a nontrivial autark assignment. Kullmann showed that a CNF is lean if and only if every clause can be used by some resolution refutation. Moreover, it is shown that a $\mathrm{CNF}$, seen as a set of clauses, can be uniquely decomposed into two subsets of clauses: the maximal autark subset and the maximal lean subset.

Although it is preferable to obtain these satisfiability preserving assignments, it is in general NP-hard to find these partial assignments. Thus, local and linear types are studied by Kullmann (2000) and Bordeaux et al. (2008), for example, where linear types are discussed in the next subsection. Local types are defined in terms of constraints. More precisely, they require a partial assignment to be satisfiability preserving for each constraint, when each constraint itself is regarded as a problem instance. We note that an autark assignment is in fact a local type, since it is defined in terms of constraints. A partial assignment is called locally satisfying if it is satisfying for each constraint, and locally fixable if it is fixable for each constraint. As seen in Section 3, the strength of locality depends on the constraint classes.

Before discussing linear autark assignments, we state two concepts related to satisfiability preserving assignments: symmetric breaking and backdoor sets. Symmetry breaking, which can be regarded as a generalization of satisfiability preserving assignments, is also widely used to reduce the search space in artificial intelligence (Freuder, 1991; Jeavons, Cohen, \& Cooper, 1994; Prestwich, 2004). A backdoor set is a subset of variables whose instantiation leads a given formula to the one which is computationally easy to solve (Crama, Ekin, \& Hammer, 1997; Williams, Gomes, \& Selman, 2003; Gaspers \& Szeider, 2012).

\subsection{Linear Autark Assignments}

Since it is NP-hard to find an autark assignment, Kullmann (2000) introduced linear autark assignments to efficiently find an autark assignment. Linear autark assignments can be seen as polyhedral conic inner approximations of autark assignments in the sense that they correspond to polyhedral cones, which are defined by systems of linear inequalities, contained 
in the set of vectors corresponding to the set of autark assignments. This implies that they can be computed in polynomial time via solving linear programming problems (Kullmann, 2000). We note that the correspondence of partial assignments and vectors is also introduced by Kullmann. Although autark assignments are not always linear, linearity can be used to efficiently find autark assignments and have a possible application to speed up heuristic SAT algorithms. Moreover, linear autark assignments provide a unifying algorithm to solve well-known tractable subclasses of the SAT problem. For example, 2-, Horn and q-Horn SAT problems are polynomially solvable by iteratively finding linear autark assignments (Kullmann, 2000; van Maaren, 2000).

At first, Kullmann (2000) introduced unweighted linear autark assignments, namely, those assignments that correspond to polyhedral cones defined by so-called clause-variable matrices of CNFs. Later, Kullmann (2003) used qualitative matrix analysis (e.g., Brualdi \& Shader, 1995) to analyze autark assignments. For example, Kullmann (2003, Lemma 3.5) shows that any matrix in the qualitative class of the clause-variable matrix, i.e., matrix that has the same sign pattern as the clause-variable matrix, defines polyhedral cones that derive autark assignments (and such an assignment is sometimes called a weighted linear autark assignment). Namely, it is shown that polyhedral cones defined by such matrices are included in the set of vectors corresponding to the autark assignments. Kullmann (2003, Lemma 3.5) also shows that any autark assignment can be obtained by linear inequalities defined by some matrix in the qualitative class. However, it is not investigated which matrices define a good polyhedral conic approximation nor can we do better if we consider general convex inner approximation, i.e., convex sets contained in the set of vectors corresponding to the autark assignments. In fact, the field of linear autark assignments appears largely unexplored as pointed out by, e.g., Kleine Büning and Kullmann (2009, Section 11.11). These motivate us to study convex inner approximations of autark assignments and especially general linear types, i.e., satisfiability preserving assignments that can be represented by general linear programming problems.

We note that connection between linear programmings and SAT is explored by Chandru and Hooker (1999). They investigate the power of a linear programming relaxation of an integer programming formulation of SAT. Their work can be seen as an investigation of a convex outer approximation of integral solutions in the sense that the feasible solution of the relaxed problem subsume that of the original one. On the other hand, we investigate a convex inner approximation of integral solutions (and satisfiability preserving assignments) in the sense that the convex sets we consider are contained in the (transformed) feasible solutions of the original problem.

\subsection{The Results in this Paper}

In this paper, we mainly deal with the Boolean CSP and investigate linear fixable, autark and satisfying partial assignments, i.e., those satisfiability preserving assignments corresponding to polyhedral cones in the associated vector space. Kullmann introduced linear autark assignments, namely, satisfiability preserving assignments that can be obtained efficiently via solving linear programming problems. These linear autark assignments correspond to

specific cones, i.e., cones defined by clause-variable matrices. It is important to consider more general cones and convex sets dominating (namely, containing) these specific cones, 
since they might be more useful in practice. We hence consider general convex inner approximations of satisfying preserving assignments. We show that, even if viewed from the generalized framework, Kullmann's framework offers good approximations of satisfiability preserving assignments.

\subsubsection{Linear Satisfiability Preserving Assignments}

We here describe our extensions of Kullmann's work to general convex sets and cones. The results stated below in this paragraph are for SAT instances, i.e., CNFs. We first show that the weighted linear autark assignments introduced by Kullmann (2003) dominate general polyhedral cones in term of the set of vectors for autark assignments (see Theorem 3 in Section 3). Namely, no matter how complex a polyhedral cone is, it is contained in a polyhedral cone defined by a matrix in the qualitative class of the clause-variable matrix. This extends the result that any autark assignment can be obtained as a weighted linear autark assignment (Kullmann, 2003, Lemma 3.5), since there always exists a polyhedral cone containing a vector corresponding to the autark assignment. Moreover, we show that the weighted linear autark assignments dominate general convex sets in terms of the set of partial assignments (see Theorem 4). Namely, for any convex set contained in the set of vectors corresponding to the autark assignments, there exists a matrix in the qualitative class of the clause-variable matrix such that the set of the partial assignments corresponding to the polyhedral cone defined by the matrix subsumes the set of the partial assignments corresponding to the convex set. Furthermore, if the input is monotone, i.e., if the input CNF contains no negative literal, then any matrix in the qualitative class of the clausevariable matrix defines a polyhedral cone that contains all the autark assignments, which shows the strength of linear autark assignments (see Theorem 5). Similar results hold for satisfying partial assignments (see Theorems 3-5). Finally, if the input is monotone and prime, then any matrix in the qualitative class of the clause-variable matrix defines a polyhedral cone that is maximal as a convex set (see Theorem 6). Here, a CNF $\varphi$ is called prime if replacing a clause $c \in \varphi$ by a proper subclause of $c$ produces a CNF which is not equivalent to $\varphi$. We note that such matrices were considered in relation with autark and satisfying partial assignment (Kullmann, 2003), however, it is not investigated in the literature how good the polyhedral cones that these matrices define are. We also note that any Boolean CSP instance can be transformed to a (possibly exponential size) CNF, and hence these results indicate how to find a good convex set for any Boolean CSP.

\subsubsection{Algorithms of Finding Linear Satisfiability Preserving Assignments for Integer Linear Systems}

We consider computing linear satisfiability preserving assignments, especially linear locally fixable assignments, of a given integer linear system. If each constraint is represented by a prime CNF, then we can compute a linear locally fixable assignment by considering a linear autark assignment, which can be easily formulated from given linear inequalities (Kullmann, 2000). Moreover, since each constraint of an integer linear system is monotone (unate, more precisely), this ensures its dominance property by Theorems 5 and 6 . However, it can be seen that a prime CNF expression is in general exponentially larger than the input. Therefore, instead of explicitly constructing the system of linear inequalities corresponding to a prime 
CNF expression $\varphi$, we solve it by using the ellipsoid method, where the separation problem is solved by using a dynamic programming similar to the one for the Knapsack Problem. Note that linear globally fixable or more general satisfiability preserving assignments cannot be easily formulated as a linear programming problem from given linear inequalities.

In this paper, we obtain the following algorithmic results. First, we provide a pseudopolynomial time algorithm to find a linear locally-fixable assignment for the $0-1$ integer linear system. Here, a 0-1 integer linear system is an integer linear system such that $D=\{0,1\}$. An algorithm is called a pseudo-polynomial time algorithm if it runs in polynomial in the length of the input and the magnitude of the largest integer occurring in the input (e.g., Garey \& Johnson, 1979, Section 4). We also show that the problem can be solved in polynomial time if every entry of the input matrix is contained in $\{0,-1,+1\}$ (see Theorem 7 in Section 4).

By extending the first result, we provide a pseudo-polynomial time algorithm to find a linear locally-fixable assignment for the (general) integer linear system (see Theorem 8 in Section 4). This is obtained by making use of the following encoding known as order encoding (Tamura, Taga, Kitagawa, \& Banbara, 2009) that transforms CSP instances with $D=\{0,1, \ldots, d\}$ to Boolean CSP instances. For each variable $x_{j}$, we take $d$ Boolean variables $x_{j 1}, \ldots, x_{j d}$ which guarantee that $x_{j}=p$ if and only if $x_{j 1}=\cdots=x_{j p}=1$ and $x_{j, p+1}=\cdots=x_{j d}=0$. We note that the number of clauses in the order encoding might be exponential in $d$ in general and hence we cannot construct the encoded CNF explicitly. Here, the ellipsoid method again copes with this difficulty.

Similar to the results for SAT by Kullmann (2000) and van Maaren (2000), we show that TVPI, Horn and q-Horn integer linear systems can be solved by repeatedly obtaining a linear locally-fixable assignment (see Theorem 9).

The rest of the paper is organized as follows. In Section 2, we formally define the CSP and introduce several (linear) satisfiability preserving assignments considered in the paper. In Section 3, we investigate the maximality of linear satisfiability preserving assignments in terms of the set of vectors and the set of partial assignments. Section 4 presents the application of linear satisfiability preserving assignments to integer linear systems.

\section{Preliminaries}

In this section, we first fix the notation to represent the constraint satisfaction problem (CSP), and define several satisfiability preserving assignments. We then introduce linear satisfiability preserving assignments, and finally recall the equivalence of optimization and separation on well-described polytopes.

\subsection{Constraint Satisfaction Problem and Satisfiability Preserving Assignments}

Let $V$ denote a set of variables with size $n=|V|$. Let $D$ denote the finite domain of the variables in $V$, where $|D| \geq 2$. For a subset $X \subseteq V$, a mapping $A: X \rightarrow D$ is called a partial assignment on $X$. We denote by $D^{X}$ the set of partial assignments on $X$. For convenience, we identify the variables and the indices in this paper. For a partial assignment $A: X \rightarrow D, X$ is denoted by $\operatorname{var}(A)$. A partial assignment $A$ is called a total assignment if $\operatorname{var}(A)=V$ holds, and called a trivial assignment if $\operatorname{var}(A)=\emptyset$ holds. For notational simplicity, we identify a partial assignment $A$ with a vector $a \in(D \cup\{*\})^{n}$ such 
that $a_{j}=A\left(x_{j}\right)$ if $x_{j} \in \operatorname{var}(A)$, and $a_{j}=*$ otherwise (i.e., $x_{j} \notin \operatorname{var}(A)$ ). For example, if $n=3$ and $A:\left\{x_{1}, x_{3}\right\} \rightarrow\{0,1,2\}$ such that $A\left(x_{1}\right)=2$ and $A\left(x_{3}\right)=0$, then we have $a=(2, *, 0)$. For an integer $k \geq 1$, a $k$-ary relation on $D$ is a subset of $D^{k}$. A constraint $C$ is a pair $C=\left\langle\left(x_{C_{1}}, \ldots, x_{C_{k}}\right), R\right\rangle$, where $x_{C_{1}}, \ldots, x_{C_{k}} \in V$ are variables and $R$ is a $k$ ary relation on $D$. $\left(x_{C_{1}}, \ldots, x_{C_{k}}\right)$ is called a scope of the constraint and $\left\{x_{C_{1}}, \ldots, x_{C_{k}}\right\}$ is written as $V(C)$. We denote by $S_{C}$ the set of the total assignments satisfying the constraint $C=\left\langle\left(x_{C_{1}}, \ldots, x_{C_{k}}\right), R\right\rangle$, that is, $S_{C}=\left\{a \in D^{n}:\left(a_{C_{1}}, \ldots, a_{C_{k}}\right) \in R\right\}$. An instance of the constraint satisfaction problem $(\mathrm{CSP})$ is defined by a triplet $(V, D, \mathcal{C})$ such that $\mathcal{C}$ denotes a set of constraints with $m=|\mathcal{C}|$. A total assignment $A \in D^{V}$ is called a solution (or a satisfying total assignment) to a CSP instance $(V, D, \mathcal{C})$ if it satisfies every constraint, i.e., $\left(a_{C_{1}}, \ldots, a_{C_{k}}\right) \in R$ holds for every constraint $\left\langle\left(x_{C_{1}}, \ldots, x_{C_{k}}\right), R\right\rangle \in \mathcal{C}$. Hence, $A \in D^{V}$ is a solution if and only if $A \in \bigcap_{C \in \mathcal{C}} S_{C}$ holds. The CSP is the problem of finding a solution to a given instance $(V, D, \mathcal{C})$.

The Boolean satisfiability problem (SAT) is regarded as a special case of the CSP, in which $V=\left\{x_{1}, \ldots, x_{n}\right\}$ denotes the set of propositional variables, i.e., $D=\{0,1\}$, and $\mathcal{C}$ is given by a conjunctive normal form $(\mathrm{CNF}) \varphi=\bigwedge_{i=1}^{m}\left(\bigvee_{j \in J_{+}^{(i)}} x_{j} \vee \bigvee_{j \in J_{-}^{(i)}} \bar{x}_{j}\right)$, where $J_{+}^{(i)}, J_{-}^{(i)} \subseteq\{1, \ldots, n\}$ and $J_{+}^{(i)} \cap J_{-}^{(i)}=\emptyset$ for all $i=1, \ldots, m$. Namely, the constraints in $\mathcal{C}$ correspond to the clauses in $\varphi$. The problem of solving an integer linear system (with finite domain) is also contained in CSPs, where each constraint is given as a linear inequality.

For a partial assignment $A$ and $X \subseteq \operatorname{var}(A)$, the restriction of $A$ to $X$, denoted by $\left.A\right|_{X}$, is defined as a partial assignment in $D^{X}$ such that $\left.A\right|_{X}(v)=A(v)$ for all $v \in X$. Let $S$ be a subset of $D^{V}$. For a partial assignment $A$, let $S[A]$ be the restriction to $V \backslash \operatorname{var}(A)$ of those elements in $S$ that are compatible with $A$, i.e., $S[A]=\left\{\left.B\right|_{V \backslash \operatorname{var}(A)}: B \in S,\left.B\right|_{\operatorname{var}(A)}=A\right\}$. For a CSP instance $(V, D, \mathcal{C})$ with the solution set $S \subseteq D^{V}$, a partial assignment $A$ is called satisfiability preserving if the satisfiability of the problem does not change after substituting it to the corresponding variables, i.e., $S[A] \neq \emptyset$ if and only if $S \neq \emptyset$. In other words, a satisfiability preserving assignment is a partial assignment which can be extended to a total assignment in $S$, if $S$ is not empty. For a CSP instance with $S=\emptyset$, any partial assignment is satisfiability preserving.

We now introduce several (globally and locally) satisfiability preserving assignments.

Definition 1 (Globally satisfiability preserving assignment). Let $(V, D, \mathcal{C})$ be a $C S P$ instance, and let $A$ be a partial assignment.

(i) $A$ is called globally satisfying for $(V, D, \mathcal{C})$ if any extension of $A$ to a total assignment is a solution.

(ii) $A$ is called globally fixable for $(V, D, \mathcal{C})$ if any solution to $(V, D, \mathcal{C})$ produces a solution by replacing those assignments to $\operatorname{var}(A)$ by $A$.

Let $S$ be a solution set to $(V, D, \mathcal{C})$. By definition, a partial assignment $A$ is globally satisfying if and only if $S[A]=D^{V \backslash \operatorname{var}(A)}$ holds. Moreover, $A$ is globally fixable if and only if $S[A] \supseteq S[B]$ holds for any partial assignment $B$ such that $\operatorname{var}(B)=\operatorname{var}(A)$.

Definition 2 (Locally satisfiability preserving assignment). Let $(V, D, \mathcal{C})$ be a $C S P$ instance, and let $A$ be a partial assignment. 
(i) $A$ is called locally satisfying for $(V, D, \mathcal{C})$ if for any constraint $C \in \mathcal{C}$, it is globally satisfying for $(V, D,\{C\})$.

(ii) $A$ is called autark for $(V, D, \mathcal{C})$ if for any constraint $C \in \mathcal{C}$ with a variable in $\operatorname{var}(A)$, it is globally satisfying for $(V, D,\{C\})$.

(iii) $A$ is called locally fixable for $(V, D, \mathcal{C})$ if for any constraint $C$, it is globally fixable for $(V, D,\{C\})$.

By definition, local fixability and autarky coincide for CNFs. See also the proof of Theorem 2 in Section 3.

Example 1. Let $\left(V=\left\{x_{1}, x_{2}, x_{3}\right\}, D=\{0,1,2\}, \mathcal{C}\right)$ be a CSP instance whose solution set $S$ is given as

$$
S=\{(0,1,1),(0,2,1),(1,1,0),(1,1,1),(1,1,2)\} .
$$

Then a partial assignment $(1,1, *)$ is globally satisfying for $(V, D, \mathcal{C})$, since $S[(1,1, *)]=$ $D^{\left\{x_{3}\right\}}$ holds. Moreover, $(0, *, 1)$ is globally fixable for $(V, D, \mathcal{C})$, since for a partial assignment $A$ with $\operatorname{var}(A)=\left\{x_{1}, x_{3}\right\}$, we have $S[A]=\{(1),(2)\}$ if $A=(0, *, 1),\{(1)\}$ if $A=(1, *, 0),(1, *, 1)$ or $(1, *, 2)$, and $\emptyset$ otherwise. We also see that $(0, *, 1)$ is not globally satisfying for $(V, D, \mathcal{C})$ and that $(1, *, 0)$ is not globally fixable for $(V, D, \mathcal{C})$.

Example 2. Let $\left(V=\left\{x_{1}, x_{2}, x_{3}\right\}, D=\{0,1,2,3\}, \mathcal{C}\right)$ be a CSP instance, where $\mathcal{C}$ is given by a linear system

$$
\begin{aligned}
2 x_{1}+3 x_{2}-x_{3} & \geq 3 \\
3 x_{1}-4 x_{3} & \geq-2 \\
-x_{2}-x_{3} & \geq-5 .
\end{aligned}
$$

As mentioned, each inequality corresponds to a constraint. We can see that the three partial assignments $(*, 1,0),(*, 2, *)$, and $(3, *, *)$ are respectively locally satisfying, autark, and locally fixable for $(V, D, \mathcal{C})$. To see that $(*, 1,0)$ is locally satisfying for $(V, D, \mathcal{C})$, we substitute it to the linear system:

$$
\begin{aligned}
2 x_{1} & \geq 0 \\
3 x_{1} & \geq-2 \\
0 & \geq-4 .
\end{aligned}
$$

Note that all inequalities in (2) are satisfied for any assignment to $x_{1}$, which implies that $(*, 1,0)$ is locally satisfying. We next show that $(*, 2, *)$ is autark for $(V, D, \mathcal{C})$. Note that $x_{2}$ is contained in the first and third constraints. We substitute it into those constraints to obtain

$$
\begin{aligned}
2 x_{1}-x_{3} & \geq-3 \\
-x_{3} & \geq-3 .
\end{aligned}
$$

Both the constraints are satisfied for any assignment to $x_{1}$ and $x_{3}$, which implies that $(*, 1,0)$ is autark. Finally, $(3, *, *)$ is locally fixable, since all the coefficients of $x_{1}$ are nonnegative in (1) and 3 is the maximum in $D$.

We remark that fixable assignments were introduced by Bordeaux et al. (2008) for single variable assignments and are also known as safe assignments in the literature (e.g., Kleine Büning \& Kullmann, 2009). The concept of autark assignments was introduced by 
Monien and Speckenmeyer (1985) for the Boolean case $D=\{0,1\}$ to construct fast exact algorithms for SAT, and was investigated by Kullmann (2000). Autark assignments for general constraints were studied by Kleine Büning and Kullmann (2009) and Kullmann (2011).

Satisfiability preserving assignments are hereditary, i.e., if a partial assignment $A$ is satisfiability preserving, then $\left.A\right|_{X}$ is also satisfiability preserving for any $X \subseteq \operatorname{var}(A)$. However, no other concepts defined above are hereditary. For example, consider a CNF $\varphi=\left(x_{1} \vee x_{2} \vee x_{3}\right)\left(\bar{x}_{1} \vee \bar{x}_{2} \vee x_{3}\right)$. Then a partial assignment $(1,0, *)$ is globally satisfying for $\varphi$. On the other hand, neither $(1, *, *)$ nor $(*, 0, *)$ is globally satisfying. Similarly, this example shows that none of the concepts such that globally fixable, locally satisfying, autark, and locally fixable assignments is hereditary.

Finally, we introduce notation to represent the sets of the satisfiability preserving assignments.

Definition 3. For a CSP instance $I$, we define $P_{\mathrm{g} \text {-sat }}(I), P_{\ell \text {-sat }}(I), P_{\text {autark }}(I), P_{\mathrm{g} \text {-fix }}(I)$, $P_{\ell \text {-fix }}(I)$ and $P_{\text {presv }}(I)$ as the set of all the globally satisfying, locally satisfying, autark, globally fixable, locally fixable and satisfiability preserving partial assignments of $I$, respectively.

\subsection{Linear Satisfiability Preserving Assignments}

In this subsection, we introduce linear satisfiability preserving assignments after reviewing several basic geometric concepts. In what follows 0 denotes the all-zero vector of appropriate size.

Let $\mathbb{R}$ denote the set of reals. A subset $Z \subseteq \mathbb{R}^{n}$ is said to be convex if $(1-\lambda) y+\lambda z \in Z$ holds for any $y, z \in Z$ and $0<\lambda<1$. A subset $Z \subseteq \mathbb{R}^{n}$ is a cone if $\lambda y \in Z$ holds for any $y \in Z$ and $\lambda>0$. For a subset $Z \subseteq \mathbb{R}^{n}$, its convex hull is the unique smallest convex set containing $Z$ and denoted by $\operatorname{conv}(Z)$. For a subset $Z \subseteq \mathbb{R}^{n}$, its conic hull is defined as $\{\lambda y: \lambda>0, y \in Z\}$. For a $y \in \mathbb{R}^{n}$, let $|y|$ denotes the Euclidean norm of $y$, i.e., $|y|=\left(y_{1}^{2}+\cdots+y_{n}^{2}\right)^{1 / 2}$. For a vector $y \in \mathbb{R}^{n}$ and a real $\varepsilon>0$, let $B(y, \varepsilon) \subseteq \mathbb{R}$ be the ball with the center $y$ and the radius $\varepsilon$, i.e., $B(y, \varepsilon)=\left\{z \in \mathbb{R}^{n}:|z-y| \leq \varepsilon\right\}$. For a subset $Z \subseteq \mathbb{R}^{n}$, its closure is defined as $\operatorname{cl}(Z)=\left\{y \in \mathbb{R}^{n}: \forall \varepsilon>0, B(y, \varepsilon) \cap Z \neq \emptyset\right\}$, and its interior is defined as $\operatorname{int}(Z)=\left\{y \in \mathbb{R}^{n}: \exists \varepsilon>0, B(y, \varepsilon) \subseteq Z\right\}$.

For two vectors $y$ and $z \in \mathbb{R}^{n}$, we write $y \geq z$, if $y_{j} \geq z_{j}$ holds for all $j=1, \ldots, n$, and $y \gg z$, if $y_{j}>z_{j}$ holds for all $j=1, \ldots, n$. A subset $Z \subseteq \mathbb{R}^{n}$ is called a (closed) polyhedron if it is the solution set of a system of linear inequalities, i.e., $Z=\left\{y \in \mathbb{R}^{n}: M y \geq b\right\}$ holds for some $m \times n$ matrix $M$ and a vector $b \in \mathbb{R}^{m}$, and is called an open polyhedron if $Z=\left\{y \in \mathbb{R}^{n}: M y \gg b\right\}$ holds for some matrix $M$ and a vector $b$. A polyhedron $Z$ that is bounded, i.e., $Z \subseteq B(0, \gamma)$ for some $\gamma>0$, is called a polytope. For a matrix $M$, let $K_{\geq}(M)=\left\{y \in \mathbb{R}^{n}: M y \geq 0\right\}$ and $K_{\gg}(M)=\left\{y \in \mathbb{R}^{n}: M y \gg 0\right\}$. A subset $Z \subseteq \mathbb{R}^{n}$ is called a (closed) polyhedral cone if $Z=K_{\geq}(M)$ holds for some matrix $M$ and open polyhedral cone if $Z=K_{\gg}(M)$ holds for some matrix $M$.

In order to define linear satisfiability preserving assignments for Boolean CSPs with $V=\left\{x_{1}, \ldots, x_{n}\right\}$ and $D=\{0,1\}$, we identify a vector $y \in \mathbb{R}^{n}$ with a partial assignment 
$a^{y} \in\{0,1, *\}^{n}$ such that

$$
a_{j}^{y}= \begin{cases}1 & \text { if } y_{j}>0 \\ 0 & \text { if } y_{j}<0 \\ * & \text { otherwise }\end{cases}
$$

We note that the $j$ th coordinate of $\mathbb{R}^{n}$ corresponds to Boolean variable $x_{j}$. We also note that this identification is introduced by Kullmann (2000). For $Z \subseteq \mathbb{R}^{n}$, let $P(Z)$ be the set of partial assignments corresponding to the vectors in $Z$, i.e., $P(Z)=\left\{a^{y}: y \in Z\right\}$. Conversely, for a set of partial assignments $P$, we define the set of vectors corresponding to $P$ by $Y(P)=\left\{y \in \mathbb{R}^{n}: a^{y} \in P\right\}$. Note that $Y(P)$ is a (not necessarily convex) cone.

Definition 4. Let $(V, D=\{0,1\}, \mathcal{C})$ be a Boolean CSP instance. A subset $P$ of globally satisfying (resp., globally fixable, locally satisfying, autark, locally fixable, and satisfiability preserving) partial assignments of $(V, D, \mathcal{C})$ is called linear if it corresponds to some polyhedral cone $K$ in $\mathbb{R}^{n}$, i.e., $P=P(K)$, and such a partial assignment in $P$ is called K-linear.

Example 3. Let $I=\left(V=\left\{x_{1}, x_{2}\right\}, D=\{0,1\}, \mathcal{C}\right)$ be a SAT instance, where $\mathcal{C}$ is given by a $C N F \varphi=x_{1} \vee x_{2}$. Then we have

$$
\begin{aligned}
& P_{\text {presv }}(I)=\{0,1, *\}^{2} \backslash\{(0,0)\}, \\
& P_{\text {autark }}(I)=P_{\ell \text {-fix }}(I)=P_{\text {g-fix }}(I)=\{(0,1),(1,0),(1,1),(1, *),(*, 1),(*, *)\}, \\
& P_{\ell \text {-sat }}(I)=P_{\text {g-sat }}(I)=\{(0,1),(1,0),(1,1),(1, *),(*, 1)\} .
\end{aligned}
$$

Consider the three polyhedral cones defined by linear systems:

$$
\begin{aligned}
& K_{1}=\left\{y \in \mathbb{R}^{2}: y_{1} \geq 0, y_{1}-y_{2} \geq 0\right\} \\
& K_{2}=\left\{y \in \mathbb{R}^{2}: y_{1}+y_{2} \geq 0\right\} \\
& K_{3}=\left\{y \in \mathbb{R}^{2}: y_{1}+y_{2}>0\right\}
\end{aligned}
$$

where we note that the inequality in the third system is strict. Then we have

$$
K_{1} \subseteq Y\left(P_{\text {presv }}(I)\right), K_{2} \subseteq Y\left(P_{\text {autark }}(I)\right) \text {, and } K_{3} \subseteq Y\left(P_{\ell \text {-sat }}(I)\right) .
$$

In fact, we have

$$
P\left(K_{1}\right) \subseteq P_{\text {presv }}(I), P\left(K_{2}\right)=P_{\text {autark }}(I) \text {, and } P\left(K_{3}\right)=P_{\ell \text {-sat }}(I) .
$$

Kullmann (2000) introduced unweighted linear autark assignments for the SAT prob$\operatorname{lem}^{1}$. Namely, for a $\operatorname{CNF} \varphi=\bigwedge_{i=1}^{m} c_{i}$, let $M_{\varphi}$ be a clause-variable matrix of $\varphi$, i.e., an $m \times n$ matrix such that $(i, j)$ entry is 1 if $x_{j} \in c_{i},-1$ if $\bar{x}_{j} \in c_{i}$, and 0 otherwise (i.e., $\left.x_{j} \notin V\left(c_{i}\right)\right)$. Then an unweighted linear autark assignment is exactly a $K_{\geq}\left(M_{\varphi}\right)$-linear autark assignment. We note that any partial assignment in $P\left(K_{\geq}\left(M_{\varphi}\right)\right)$ is autark. Kullmann (2003) implicitly studied unweighted linear globally satisfying partial assignments, which

1. More precisely, Kullmann referred to unweighted linear autark assignments as simple linear autark assignments, and partial assignments defined by iteratively applying simple linear autark assignments as linear autark assignments. 
are $K_{\gg}\left(M_{\varphi}\right)$-linear globally satisfying partial assignments. We again note that any partial assignment in $P\left(K_{\gg}\left(M_{\varphi}\right)\right)$ is globally satisfying.

Kullmann (2003) then introduced weighted linear autark and globally satisfying partial assignments. For a real matrix $M$, let $\mathcal{Q}(M)$ denote the set of matrices $N$ with its sign pattern identical to $M$, i.e., $N_{i j}<0$ (resp., $=0,>0$ ) if and only if $M_{i j}<0$ (resp., $=0$, $>0) . \mathcal{Q}(M)$ is known as the qualitative class of matrix $M$ (Brualdi \& Shader, 1995). Then a set $P$ of partial assignments is called weighted linear autark if we have $P=P\left(K_{\geq}(M)\right)$ for an $M \in \mathcal{Q}\left(M_{\varphi}\right)$, and weighted linear globally satisfying if we have $P=P\left(K_{\gg}(M)\right)$ for an $M \in \mathcal{Q}\left(M_{\varphi}\right)$. Kullmann characterizes autark and globally satisfying partial assignments in terms of the qualitative class of the clause-variable matrix of a CNF. Namely, it is shown that $P_{\text {autark }}=\bigcup_{M \in \mathcal{Q}\left(M_{\varphi}\right)} P\left(K_{\geq}(M)\right)$ and $P_{\text {g-sat }}=\bigcup_{M \in \mathcal{Q}\left(M_{\varphi}\right)} P\left(K_{\gg}(M)\right)$. We give more detailed analyses in Section 3.

We here emphasize that (un)weighted linear autark and globally satisfying partial assignments introduced by Kullmann are special cases of linear autark and globally satisfying partial assignments introduced in this paper. However, it turns out that they provide good convex inner approximations for autark and globally satisfying partial assignments; see Section 3 for more details.

Linear satisfiability preserving assignments for non-Boolean CSPs are defined via transformations to Boolean CSPs, which can be found in Section 4.

\subsection{Optimization and Separation}

We here briefly summarize the relationship between optimization and separation via the ellipsoid method (e.g., Grötschel, Lovász, \& Schrijver, 1993), which will be used in Section 4 .

Let $\mathbb{Q}$ denote the set of rationals.

Problem 1 (Optimization Problem for Polytopes). Given a polytope $Z \subseteq \mathbb{R}^{n}$ and a vector $\alpha \in \mathbb{Q}^{n}$, either

(i) assert that $Z$ is empty, or

(ii) find a vector $z \in Z$ that maximizes $\alpha y$ over $Z$.

Problem 2 (Separation Problem for Polytopes). Given a polytope $Z \subseteq \mathbb{R}^{n}$ and a vector $z \in \mathbb{Q}^{n}$, decide whether $z \in Z$, and if not, find a hyperplane that separates $z$ from $Z$; more exactly, find a vector $\alpha \in \mathbb{Q}^{n}$ such that $\alpha z>\max \{\alpha y: y \in Z\}$.

For a polytope $Z \subseteq \mathbb{R}^{n}$ and a positive integer $\ell, Z$ has facet-complexity at most $\ell$ if there exists a system of inequalities with rational coefficients that has solution set $Z$ and such that the encoding length of each inequality of the system is at most $\ell$. A well-described polytope is a triple $(Z ; n, \ell)$ where $Z \subseteq \mathbb{R}^{n}$ is a polytope with facet complexity at most $\ell$.

Theorem 1 (Grötschel et al., 1993, Thm. 6.4.9). Each of the strong optimization and separation problems can be solved in oracle-polynomial time for any well-described polytope given by an oracle for the other problem. 


\section{Properties of Satisfiability Preserving Assignments for the Boolean CSP}

In this section, we restrict our attention to the Boolean CSP and discuss several fundamental properties of satisfiability preserving assignments. The main results of the section are summarized as follows.

- The inclusion relationships between satisfiability preserving assignments including their local variants (Subsection 3.1).

- Basic properties of the set of the vectors corresponding to satisfiability preserving assignments (Subsection 3.2).

- Maximality of weighted linear satisfiability preserving assignments defined by matrices in the qualitative classes of the clause-variable matrices (Subsections 3.3 and 3.4).

From these results we can define a large family of linear satisfiability preserving assignments, from which we can compute in (pseudo-)polynomial time a satisfiability preserving assignment for integer linear system; see Section 4.

\subsection{Relationships between Satisfiability Preserving Assignments}

In this subsection, we summarize in Theorem 2 the inclusion relationships between several satisfiability preserving assignments. Recall that a $\operatorname{CNF} \varphi$ is prime if replacing a clause $c \in \varphi$ by a proper subclause of $c$ produces a CNF which is not equivalent to $\varphi$.

Theorem 2. Let I be a CSP instance. Then the following relations hold.

$$
P_{\ell \text {-sat }}(I)=P_{\text {g-sat }}(I) \subseteq P_{\text {autark }}(I) \subseteq P_{\ell \text {-fix }}(I) \subseteq P_{\text {g-fix }}(I) \subseteq P_{\text {presv }}(I)
$$

Moreover, if the constraints are represented by a CNF, then we have

$$
P_{\text {autark }}(I)=P_{\ell \text {-fix }}(I),
$$

and if the constraints are represented by a prime CNF, then we have

$$
P_{\ell-\text { fix }}(I)=P_{\text {g-fix }}(I) \text {. }
$$

By Theorem 2, in general, satisfiability preserving assignments considered in this paper form a total preorder, where a binary relation is a preorder if it is reflexive and transitive, and in addition total if any two elements are comparable. In the preorder, global fixability is the largest notion of satisfiability preserving assignments and local satisfiability (i.e., satisfiability) is the smallest one. Moreover, autarky and local fixability coincide if the constraints are given by a CNF, and local fixability and global fixability coincide if the constraints are given by a prime CNF. Hence, autarky, local fixability, and global fixability all coincide in a prime $\mathrm{CNF}$.

We note that a part of Theorem 2 is folklore in the CSP community and are obtained from basic observations. However, we cannot find this from any textbook, and it is important to know it for the following reasons. 
In order to reduce the problem size by using satisfiability preserving assignment, it is desired to find a satisfiability preserving assignment in the largest class: a globally fixable assignment in general or in CNFs, and a globally fixable (i.e., locally fixable and autark) assignment in prime CNFs. You might think that it is nonsense to deal with prime CNF instances, since we can easily see satisfiability or unsatisfiability of the instance if it is represented by a prime CNF. However, it will be used in Section 4 to compute a linear locally fixable assignment of an integer linear system by implicitly constructing a prime CNF expression for each linear inequality.

Before proving the theorem, we show by the following examples that no other relation than (9) in Theorem 2 holds for the general CSP.

Example 4. Consider the following four Boolean CSP instances $I_{i}=\left(V, D, \mathcal{C}_{i}\right)(i=$ $1,2,3,4)$ with two variables, i.e., $V=\left\{x_{1}, x_{2}\right\}$ and $D=\{0,1\}$.

(i) Let $\mathcal{C}_{1}$ be given as a CNF $\varphi=x_{1} x_{2}$, namely, $\mathcal{C}_{1}=\left\{\left\langle\left(x_{1}\right),\{(1)\}\right\rangle,\left\langle\left(x_{2}\right),\{(1)\}\right\rangle\right\}$. Then $(1, *)$ is contained in $P_{\text {autark }}\left(I_{1}\right)$, but not in $P_{\mathrm{g} \text {-sat }}\left(I_{1}\right)$. Indeed, $(1, *)$ is satisfying for the first constraint, and the second constraint does not contain variable $x_{1}$ that is fixed by $(1, *)$. Hence, it is autark. On the other hand, it is not globally satisfying for $I_{1}$, since $(1,0)$ is not a solution to $I_{1}$.

(ii) Let $\mathcal{C}_{2}$ be given as a single inequality $x_{1}+x_{2} \geq 2$, namely, $\mathcal{C}_{2}=\left\{\left\langle\left(x_{1}, x_{2}\right),\{(1,1)\}\right\rangle\right\}$. Then $(1, *)$ is contained in $P_{\ell-\text { fix }}\left(I_{2}\right)$, but not in $P_{\text {autark }}\left(I_{2}\right)$. Indeed, for the solution set $S=\{(1,1)\}$ to the unique constraint, we have $S[(1, *)] \supseteq S[(0, *)]$ and thus $(1, *)$ is locally fixable. However, it is not autark, since $(1,0)$ is not contained in $S$.

(iii) Let $\mathcal{C}_{3}$ be given as a $C N F \varphi=x_{1}\left(x_{1} \vee x_{2}\right)$, namely, $\mathcal{C}_{3}=\left\{\left\langle\left(x_{1}\right),\{(1)\}\right\rangle,\left\langle\left(x_{1}, x_{2}\right),\{(0,1)\right.\right.$, $(1,0),(1,1)\}\rangle\}$. Then $(*, 0)$ is contained in $P_{\text {g-fix }}\left(I_{3}\right)$, but not in $P_{\ell-\text { fix }}\left(I_{3}\right)$. Indeed, for the solution set $S=\{(1,0),(1,1)\}$ to the instance, we have $S[(*, 0)] \supseteq S[(*, 1)]$ and thus $(*, 0)$ is globally fixable. However, it is not locally fixable, since $S^{\prime}[(*, 0)] \nsupseteq$ $S^{\prime}[(*, 1)]$ holds for the solution set $S^{\prime}$ to the second constraint.

(iv) Let $\mathcal{C}_{4}$ be given as a $C N F \varphi=\left(x_{1} \vee x_{2}\right)$, namely, $\mathcal{C}_{4}=\left\{\left\langle\left(x_{1}, x_{2}\right),\{(0,1),(1,0),(1,1)\}\right\rangle\right\}$. Then $(0, *)$ is contained in $P_{\text {presv }}\left(I_{4}\right)$, but not in $P_{\mathrm{g} \text {-fix }}\left(I_{4}\right)$. Indeed, $S[(0, *)]=\{(1)\} \neq$ $\emptyset$ holds for the solution set $S$ to the instance and hence it is satisfiability preserving. However, it is not globally fixable, since $S[(0, *)] \nsupseteq S[(1, *)]$ holds.

Proof of Theorem 2. We first show (9). It is not difficult from the definitions that

$$
P_{\ell \text {-sat }}(I)=P_{\text {g-sat }}(I) \subseteq P_{\text {autark }}(I) \text { and } P_{\text {g-fix }}(I) \subseteq P_{\text {presv }}(I) .
$$

For $P_{\text {autark }}(I) \subseteq P_{\ell \text {-fix }}(I)$, let $A$ be an autark assignment. If $\operatorname{var}(A) \cap V(C) \neq \emptyset$ for a constraint $C$, then $S_{C}[A]\left(=D^{V \backslash \operatorname{var}(A)}\right)$ includes $S_{C}[B]$ for any $B \in D^{\operatorname{var}(A)}$. On the other hand, if $\operatorname{var}(A) \cap V(C)=\emptyset$ for a constraint $C$, then $S_{C}[A]=S_{C}[B]$ for any $B \in D^{\operatorname{var}(A)}$. Thus, $A$ is locally fixable and we have $P_{\text {autark }}(I) \subseteq P_{\ell \text {-fix }}(I)$.

For $P_{\ell \text {-fix }}(I) \subseteq P_{\text {g-fix }}(I)$, let $A$ be a locally fixable assignment and $S$ be the solution set of $I$. Recall that $S=\bigcap_{C \in \mathcal{C}} S_{C}$ holds. Hence we have $S[A]=\bigcap_{C \in \mathcal{C}} S_{C}[A]$. Since for any $B \in D^{\operatorname{var}(A)}$ we have $S_{C}[A] \supseteq S_{C}[B]$, it holds that $S[A]=\bigcap_{C \in \mathcal{C}} S_{C}[A] \supseteq \bigcap_{C \in \mathcal{C}} S_{C}[B]=$ $S[B]$. Hence $A$ is globally fixable.

We then show (10). Let $\varphi$ be a CNF and $A$ be a locally fixable assignment. For a clause $c \in \varphi$ with $V(c) \cap \operatorname{var}(A) \neq \emptyset$, there exists a partial assignment $B$ such that $c[B]=D^{V \backslash \operatorname{var}(A)}$ 
holds. Since local fixability of $A$ implies that $c[A] \supseteq c[B]$ holds, we have $c[A]=D^{V \backslash \operatorname{var}(A)}$. Hence $A$ is autark.

We finally show (11). Let $\varphi$ be a prime CNF that represents a Boolean CSP instance, and let $A$ be a partial assignment. Assuming that $A$ is not locally fixable, we show that it is not globally fixable.

Let $S$ be the set of the solutions of $\varphi$. Since $A$ is not locally fixable, there exists a clause $c$ in $\varphi$ such that $S_{c}[A] \nsupseteq S_{c}[B]$ for some partial assignment $B$. This implies that $A$ is not a satisfying partial assignment for $c$, i.e., $S_{c}[A] \neq\{0,1\}^{V \backslash \operatorname{var}(A)}$. Then, by primality of $\varphi$, there exists a globally satisfying total assignment $A^{*}$ for $\varphi$ that does not satisfy $S_{c}[A]$, namely, $\left.A^{*}\right|_{V \backslash \operatorname{var}(A)} \notin S_{C}[A]$. This implies that $\left.A^{*}\right|_{V \backslash \operatorname{var}(A)} \notin S[A]$. Since $\left.A^{*}\right|_{V \backslash \operatorname{var}(A)} \in S\left[\left.A^{*}\right|_{\operatorname{var}(A)}\right]$, we have $S[A] \nsupseteq S\left[\left.A^{*}\right|_{\operatorname{var}(A)}\right]$. Hence $A$ is not globally fixable for $S$.

Since $P_{\text {g-sat }}=P_{\ell \text {-sat }}$ holds for the general Boolean CSP in Theorem 2, we simply write $P_{\text {sat }}$, and also write a satisfying partial assignment without having "globally" nor "locally."

Let us finally mention integer linear systems. Note that any CNF can be represented by a 0-1 integer linear system, where we recall that a 0-1 integer linear system is an integer linear system with $D=\{0,1\}$. This is because a $0-1$ assignment satisfies a clause $c=$ $\left(\bigvee_{j \in J_{+}} x_{j} \vee \bigvee_{j \in J_{-}} \bar{x}_{j}\right)$ if and only if it satisfies $\sum_{j \in J_{+}} x_{j}+\sum_{j \in J_{-}}\left(1-x_{j}\right) \geq 1$. Thus it follows from Example 4 that there exists a 0-1 integer linear system such that either (10) or (11) does not hold.

\subsection{Characterizing $Y\left(P_{\text {presv }}(I)\right)$ and $Y\left(P_{\text {sat }}(I)\right)$ for Boolean CSP Instance $I$}

Let $I$ be an instance of the Boolean CSP, and let $S$ be the solution set of $I$. We characterize $Y\left(P_{\text {presv }}(I)\right)$ and $Y\left(P_{\text {sat }}(I)\right)$ in terms of interior and closure of $Y(S)$. We first explain the relation between $S$ and $Y(S)$ where $I$ is given by a CNF. Let us see $Y(S), \operatorname{cl}(Y(S))$, and $\operatorname{int}(\operatorname{cl}(Y(S)))$. Recall that $n$ is the number of the variables. We write $S(I)$ as the solution set of a CSP instance $I$.

Example 5. Consider the three Boolean CSP instances $I_{i}=\left(V=\left\{x_{1}, x_{2}\right\}, D=\{0,1\}, \mathcal{C}_{i}\right)$ $(i=1,2,3)$, where $I_{1}$ is given by a literal $x_{1}, I_{2}$ by a clause $c=\left(\bar{x}_{1} \vee x_{2}\right)$, and $I_{3}$ by a $C N F$ $\varphi=x_{1} \wedge c$. Let us see the difference between $Y(S), \operatorname{cl}(Y(S))$, and $\operatorname{int}(\operatorname{cl}(Y(S)))$.

$$
\begin{aligned}
Y\left(S\left(I_{1}\right)\right) & =\left\{y \in \mathbb{R}^{2}:\left(y_{1}>0 \text { and } y_{2}>0\right) \text { or }\left(y_{1}>0 \text { and } y_{2}<0\right)\right\}, \\
\operatorname{cl}\left(Y\left(S\left(I_{1}\right)\right)\right) & =\left\{y \in \mathbb{R}^{2}: y_{1} \geq 0\right\}, \text { and } \\
\operatorname{int}\left(\operatorname{cl}\left(Y\left(S\left(I_{1}\right)\right)\right)\right) & =\left\{y \in \mathbb{R}^{2}: y_{1}>0\right\} .
\end{aligned}
$$

From the above example, we can see that $Y(S)$ only contains the vectors corresponding to the solutions and hence we do not have the vectors corresponding to partial assignments. By taking the closure of $Y(S)$, we obtain the vector corresponding to partial assignments, and in fact we have $\operatorname{cl}(Y(S))=Y\left(P_{\text {presv }}\right)$. However, it also contains vectors not corresponding to satisfying partial assignments. By further taking the interior, we finally get the vectors that correspond to satisfying partial assignments. Namely, we have $\operatorname{int}(\operatorname{cl}(Y(S)))=Y\left(P_{\text {sat }}\right)$. We will show that these equations hold for the general Boolean CSP in the following. 
We also have

$$
\begin{aligned}
Y\left(S\left(I_{2}\right)\right) & =\left\{y \in \mathbb{R}^{2}:\left(y_{1}<0 \text { and } y_{2}>0\right), \quad\left(y_{1}<0 \text { and } y_{2}<0\right),\right. \\
\left.\operatorname{or}\left(y_{1}>0 \text { and } y_{2}>0\right)\right\}, & \\
\operatorname{cl}\left(Y\left(S\left(I_{2}\right)\right)\right) & =\left\{y \in \mathbb{R}^{2}: y_{1} \leq 0 \text { or } y_{2} \geq 0\right\}, \text { and } \\
\operatorname{int}\left(\operatorname{cl}\left(Y\left(S\left(I_{2}\right)\right)\right)\right) & =\left\{y \in \mathbb{R}^{2}: y_{1}<0 \text { or } y_{2}>0\right\},
\end{aligned}
$$

and

$$
\begin{aligned}
Y\left(S\left(I_{3}\right)\right) & =\left\{y \in \mathbb{R}^{2}: y_{1}>0 \text { and } y_{2}>0\right\}, \\
\operatorname{cl}\left(Y\left(S\left(I_{3}\right)\right)\right) & =\left\{y \in \mathbb{R}^{2}: y_{1} \geq 0 \text { and } y_{2} \geq 0\right\}, \text { and } \\
\operatorname{int}\left(\operatorname{cl}\left(Y\left(S\left(I_{3}\right)\right)\right)\right) & =\left\{y \in \mathbb{R}^{2}: y_{1}>0 \text { and } y_{2}>0\right\} .
\end{aligned}
$$

Note that these examples show that $Y(S), \operatorname{cl}(Y(S))$ and/or $\operatorname{int}(\operatorname{cl}(Y(S)))$ might not be convex in general.

In the above example, we have $\operatorname{cl}\left(Y\left(S\left(I_{1}\right) \cap S\left(I_{2}\right)\right)\right) \neq \operatorname{cl}\left(Y\left(S\left(I_{1}\right)\right)\right) \cap \operatorname{cl}\left(Y\left(S\left(I_{2}\right)\right)\right)$. Therefore, we cannot compute $\operatorname{cl}(Y(S))$ by simply taking the intersection of the closures of the solutions to each constraint.

We now characterize satisfiability preserving and satisfying partial assignments of the Boolean CSP.

Proposition 1. Let $I=(V, D=\{0,1\}, \mathcal{C})$ be a Boolean CSP instance with solution set $S$, i.e., the set of satisfying total assignments. Then we have the following statements.

(i) If $S \neq \emptyset$, then $Y\left(P_{\text {presv }}(I)\right)=\operatorname{cl}(Y(S))$.

(ii) $Y\left(P_{\text {sat }}(I)\right)=\operatorname{int}(\operatorname{cl}(Y(S)))$.

Proof. (i): For $Y\left(P_{\text {presv }}(I)\right) \subseteq \operatorname{cl}(Y(S))$, suppose that $y$ is not contained in $\operatorname{cl}(Y(S))$. We show that $y \notin Y\left(P_{\text {presv }}\right)$, i.e., $a^{y}$ is not satisfiability preserving.

Since $y \notin \operatorname{cl}(Y(S))$, there exists an $\varepsilon>0$ such that $B(y, \varepsilon) \cap \operatorname{cl}(Y(S))=\emptyset$. Thus, for sufficiently small $\varepsilon^{\prime}>0$, we have $y+\varepsilon^{\prime} w \notin Y(S)$ and $a_{j}^{y+\varepsilon^{\prime} w}=a_{j}^{y}\left(j \in \operatorname{var}\left(a^{y}\right)\right)$ for all $w \in\{-1,1\}^{n}$. This implies that $a^{y}$ is not extendable to any satisfying total assignment. Hence, $a^{y}$ is not satisfiability preserving.

For $Y\left(P_{\text {presv }}(I)\right) \supseteq \operatorname{cl}(Y(S))$, suppose that $y$ is contained in $\operatorname{cl}(Y(S))$. Then for sufficiently small $\varepsilon>0, y+\varepsilon z \in Y(S)$ and $a_{j}^{y+\varepsilon z}=a_{j}^{y}\left(j \in \operatorname{var}\left(a^{y}\right)\right)$ hold for some $z \in B(0,1)$. Thus, $a^{y}$ is extendable to a satisfying total assignment, implying that $a^{y}$ is satisfiability preserving.

(ii): If $S=\emptyset$, then there exits no satisfying partial assignment. Thus, $Y\left(P_{\text {sat }}(I)\right)=\emptyset$. We also have $Y(S)=\emptyset$ and thus $\operatorname{int}(\operatorname{cl}(Y(S)))=\emptyset$. Hence (ii) holds.

We then assume that $S \neq \emptyset$. For $Y\left(P_{\text {sat }}(I)\right) \subseteq \operatorname{int}(\operatorname{cl}(Y(S)))$, assume that $y \in Y\left(P_{\text {sat }}(I)\right)$, i.e., $a^{y}$ is satisfying. Let $\varepsilon$ be a positive real such that $\varepsilon<\left|y_{j}\right|$ for any $y_{j} \neq 0$. We show that $B(y, \varepsilon) \subseteq \operatorname{cl}(Y(S))$, which implies that $y \in \operatorname{int}\left(\operatorname{cl}(Y(S))\right.$. Let $y^{\prime} \in B(y, \varepsilon)$ and let $\varepsilon^{\prime}>0$. We have to show that $B\left(y^{\prime}, \varepsilon^{\prime}\right) \cap Y(S) \neq \emptyset$ holds. Define $z \in B(0,1)$ as $\left|z_{j}\right|<\left(\left|y_{j}\right|-\varepsilon\right) / \varepsilon^{\prime}$ if $y_{j} \neq 0, z_{j} / y_{j}^{\prime}>0$ if $y_{j}=0$ and $y_{j}^{\prime} \neq 0$, and $z_{j}>0$ otherwise (i.e., $y_{j}=y_{j}^{\prime}=0$ ). Then we have $a_{j}^{y^{\prime}+\varepsilon^{\prime} z}=a_{j}^{y}(j \in \operatorname{var}(A))$ and $\operatorname{var}\left(a^{y^{\prime}+\varepsilon^{\prime} z}\right)=V$. Since $a^{y}$ is satisfying, $a^{y^{\prime}+\varepsilon^{\prime} z}$ is a satisfying total assignment, i.e., a solution. Hence we have $B\left(y^{\prime}, \varepsilon^{\prime}\right) \cap Y(S) \neq \emptyset$.

For $Y\left(P_{\text {sat }}(I)\right) \supseteq \operatorname{int}(\operatorname{cl}(Y(S)))$, suppose that $y$ is contained in the interior of the closure of $Y(S)$. Then for any $w \in\{-1,1\}^{n}$, by taking sufficiently small $\varepsilon>0$, we have $y+\varepsilon w \in$ 
$\operatorname{int}(\operatorname{cl}(Y(S))), a_{j}^{y+\varepsilon w}=a_{j}^{y}(j \in \operatorname{var}(A))$ and $\operatorname{var}\left(a^{y+\varepsilon w}\right)=V$ (i.e., $(y+\varepsilon w)_{j} \neq 0$ for all $\left.j\right)$. Note that $\operatorname{cl}(Y(S)) \cap Y\left(\{0,1\}^{V} \backslash S\right)=\emptyset$. Hence $y+\varepsilon w \in \operatorname{int}(\operatorname{cl}(Y(S)))$ and $(y+\varepsilon w)_{j} \neq 0$ for all $j$ imply that $y+\varepsilon w \in Y(S)$. Therefore $a^{y+\varepsilon w}$ is a satisfying total assignment. Thus, $a^{y}$ is arbitrarily extendable to a satisfying total assignment and hence it is satisfying.

If $S=\emptyset$, then (i) does not hold, since every partial assignment is satisfiability preserving, i.e., $Y\left(P_{\text {presv }}(I)\right)=\mathbb{R}^{n}$, and $\operatorname{cl}(Y(S))=\emptyset$, implying that $Y\left(P_{\text {presv }}(I)\right) \neq \operatorname{cl}(Y(S))$.

Proposition 1 shows that $Y\left(P_{\text {presv }}\right)$ and $Y\left(P_{\text {sat }}\right)$ can be characterized by $Y(S)$.

In this paper, we consider the vector space corresponding to partial assignments. Note that we can obtain a satisfiability preserving or satisfying partial assignments by finding a point in $Y\left(P_{\text {presv }}\right)$ or $Y\left(P_{\text {sat }}\right)$. However, these sets are non-convex in general and it is hard to find a point in these sets. We thus consider convex inner approximations of these sets, where convexity helps to find a point in these sets. We want to define "large" convex inner approximations, and hence investigate maximal convex inner approximations in the following two subsections.

\subsection{Maximality of Weighted Linear Satisfiability Preserving Assignments for General CNFs}

We here investigate convex inner approximation of satisfiability preserving assignments for general CNFs. Before stating the main results of this subsection, we state several auxiliary results (Propositions 2-5).

The following proposition shows that we have only to consider convex cones to find large convex subsets in terms of the set of vectors.

Proposition 2. Let $I=(V, D=\{0,1\}, \mathcal{C})$ be a Boolean CSP instance.

(i) For a closed subset $Z \subseteq Y\left(P_{\text {presv }}(I)\right)$, there exists a closed cone $Z^{*}$ such that $Z \subseteq$ $Z^{*} \subseteq Y\left(P_{\text {presv }}(I)\right)$. If $Z$ is in addition convex (resp., polyhedral), then $Z^{*}$ can be taken from convex (resp., polyhedral) cones.

(ii) For an open subset $Z \subseteq Y\left(P_{\text {sat }}(I)\right)$, there exists an open cone $Z^{*}$ such that $Z \subseteq Z^{*} \subseteq$ $Y\left(P_{\text {sat }}(I)\right)$. If $Z$ is in addition convex (resp., polyhedral), then $Z^{*}$ can be taken from convex (resp., polyhedral) cones.

Proof. (i): Let $Z^{*}$ be the closure of the conic hull of $Z$. Then we have $Z^{*} \subseteq Y\left(P_{\text {presv }}(I)\right)$, since $Y\left(P_{\text {presv }}(I)\right)$ is a closed cone by Proposition 1. It is well known that convexity and polyhedrality are preserved by taking the closure of the conic hull (e.g., Rockafellar, 1970, Thm. 19.7).

(ii): Let $Z^{*}$ be the conic hull of $Z$. Then $Z^{*}$ is open, and we have $Z^{*} \subseteq Y\left(P_{\text {sat }}(I)\right)$, since $Y\left(P_{\text {sat }}(I)\right)$ is a cone. Since convexity and polyhedrality are preserved by taking the conic hull, the proof is completed.

By Proposition 1, $Y\left(P_{\text {presv }}(I)\right)$ and $Y\left(P_{\text {sat }}(I)\right)$ are closed and open, respectively. Hence, the proposition above restricts attention to closed and open subsets of $Y\left(P_{\text {presv }}(I)\right)$ and $Y\left(P_{\text {sat }}(I)\right)$, respectively. We note that the closedness condition of $Z$ in (i) is not necessary.

We then show the following proposition on the clause-variable matrix.

Proposition 3. Let $I=(V, D=\{0,1\}, \mathcal{C})$ be a SAT instance, where $\mathcal{C}$ is given by a $C N F$ $\varphi$. Then we have 
(i) $\operatorname{cl}\left(K_{\gg}\left(M_{\varphi}\right)\right) \subseteq K_{\geq}\left(M_{\varphi}\right) \subseteq Y\left(P_{\text {autark }}(I)\right) \subseteq Y\left(P_{\text {presv }}(I)\right)$,

(ii) $K_{\gg}\left(M_{\varphi}\right) \subseteq Y\left(P_{\text {sat }}(I)\right)$.

Proof. It is known by Kullmann that $K_{\geq}\left(M_{\varphi}\right)$ is contained in $Y\left(P_{\text {autark }}(I)\right.$ ) (Kullmann, 2000, Lemma 4.9) and $K_{\gg}\left(M_{\varphi}\right)$ is contained in $Y\left(P_{\text {sat }}(I)\right)$ (Kullmann, 2003, Lemma 3.2). Moreover, we have $K_{\gg}\left(M_{\varphi}\right) \subseteq K_{\geq}\left(M_{\varphi}\right)$ by definition, and since taking the closure preserves the inclusion relation, we have $\operatorname{cl}\left(K_{\gg}\left(M_{\varphi}\right)\right) \subseteq \operatorname{cl}\left(K_{\geq}\left(M_{\varphi}\right)\right)=K_{\geq}\left(M_{\varphi}\right)$. The last equality holds since $K_{\geq}\left(M_{\varphi}\right)$ is a closed set. Finally, by Theorem 2 , we have $Y\left(P_{\text {autark }}(I)\right) \subseteq$ $Y\left(P_{\text {presv }}(I)\right)$.

We note that $\operatorname{cl}\left(K_{\gg}\left(M_{\varphi}\right)\right) \nsubseteq Y\left(P_{\text {sat }}(I)\right)$ and/or $\operatorname{cl}\left(K_{\gg}\left(M_{\varphi}\right)\right) \neq K_{\geq}\left(M_{\varphi}\right)$ might hold in general. Let $I_{1}=\left(V_{1}=\left\{x_{1}\right\}, D=\{0,1\}, F_{1}\right)$, where $\mathcal{C}_{1}$ is given by $\varphi_{1}=x_{1}$, and $I_{2}=\left(V_{2}=\left\{x_{1}, x_{2}\right\}, D=\{0,1\}, F_{2}\right)$, where $\mathcal{C}_{2}$ is given by $\varphi_{2}=\left(x_{1} \vee \bar{x}_{2}\right)\left(\bar{x}_{1} \vee x_{2}\right)$. Then we have $\operatorname{cl}\left(K_{\gg}\left(M_{\varphi_{1}}\right)\right)=\left\{y_{1} \in \mathbb{R}: y_{1} \geq 0\right\}$ and $Y\left(P_{\text {sat }}\left(I_{1}\right)\right)=\left\{y_{1} \in \mathbb{R}: y_{1}>0\right\}$, which implies that $\operatorname{cl}\left(K_{\gg}\left(M_{\varphi_{1}}\right)\right) \nsubseteq Y\left(P_{\text {sat }}\left(I_{1}\right)\right)$ holds. Also, we have $\operatorname{cl}\left(K_{\gg}\left(M_{\varphi_{2}}\right)\right)=\emptyset$ and $K_{\geq}\left(M_{\varphi_{2}}\right)=\left\{y \in \mathbb{R}^{2}: y_{1}=y_{2}\right\}$, implying that $\operatorname{cl}\left(K_{\gg}\left(M_{\varphi_{2}}\right)\right) \neq K_{\geq}\left(M_{\varphi_{2}}\right)$ holds.

By Proposition 3, an autark assignment $A$ is called unweighted linear if $A \in P\left(K_{\geq}(M)\right)$ holds, and a satisfying partial assignment $A$ is called unweighted linear if $A \in P\left(K_{\gg}(M)\right)$ holds.

Example 6. Let $\left(V=\left\{x_{1}, x_{2}, x_{3}\right\}, D=\{0,1\}, \mathcal{C}\right)$ be a SAT instance, where $\mathcal{C}$ is given by a CNF $\varphi=\left(\bar{x}_{1} \vee x_{2}\right)\left(x_{1} \vee x_{3}\right)\left(x_{2} \vee x_{3}\right)$. Then an autark assignment $(*, *, 1)$ and a satisfying partial assignment $(1,1, *)$ are unweighted linear, since $(0,0,1) \in K_{\geq}\left(M_{\varphi}\right)$ and $(1,2,0) \in K_{\gg}\left(M_{\varphi}\right)$ hold.

We note that $K_{\geq}\left(M_{\varphi}\right)=\{0\}$ and $K_{\gg}\left(M_{\varphi}\right)=\emptyset$ might hold in general as the next example shows.

Example 7. Let $I=\left(V=\left\{x_{1}, x_{2}, x_{3}\right\}, D=\{0,1\}, \mathcal{C}\right)$ be a SAT instance, where $\mathcal{C}$ is given by a $C N F \varphi=\left(x_{1} \vee x_{2} \vee x_{3}\right)\left(x_{1} \vee \bar{x}_{2} \vee \bar{x}_{3}\right)\left(\bar{x}_{1} \vee x_{2} \vee \bar{x}_{3}\right)\left(\bar{x}_{1} \vee \bar{x}_{2} \vee x_{3}\right)$. It can be verified that

$$
\begin{aligned}
& P_{\text {presv }}(I)=\{0,1, *\}^{3} \backslash\{(0,0,0),(0,1,1),(1,0,1),(1,1,0)\}, \\
& P_{\text {autark }}(I)=\{(0,0,1),(0,1,0),(1,0,0),(1,1,1),(*, *, *)\}, \\
& P_{\text {sat }}(I)=\{(0,0,1),(0,1,0),(1,0,0),(1,1,1)\} .
\end{aligned}
$$

On the other hand, there exists no nontrivial unweighted linear autark assignment nor unweighted linear satisfying partial assignment. We note that

$$
M_{\varphi}=\left(\begin{array}{rrr}
1 & 1 & 1 \\
1 & -1 & -1 \\
-1 & 1 & -1 \\
-1 & -1 & 1
\end{array}\right),
$$

and hence $K_{\geq}\left(M_{\varphi}\right)=\{0\}$ and $K_{\gg}\left(M_{\varphi}\right)=\emptyset$ hold. Indeed, summing the inequalities $y_{1}+$ $y_{2}+y_{3} \geq 0$ and $y_{1}-y_{2}-y_{3} \geq 0$ in $M_{\varphi} y \geq 0$ yields $y_{1} \geq 0$. Similarly, from $-y_{1}+y_{2}-y_{3} \geq 0$ and $-y_{1}-y_{2}+y_{3} \geq 0$, we obtain $-y_{1} \geq 0$. This implies $y_{1}=0$. We can obtain $y_{2}=y_{3}=$ 0 in a similar way. Hence, we have $K_{\geq}\left(M_{\varphi}\right)=\{0\}$. By replacing the inequalities by strict inequalities in the above argument, we obtain $y_{1}>0$ and $y_{1}<0$. Hence, we have $K_{\gg}\left(M_{\varphi}\right)=\emptyset$. 
We then consider weighted linear satisfiability preserving assignments for a $\operatorname{CNF} \varphi$, i.e., partial assignments defined by some matrix $M$ in $\mathcal{Q}\left(M_{\varphi}\right)$ or $\mathcal{Q}_{0}\left(M_{\varphi}\right)$. Here, $\mathcal{Q}_{0}(M)$ denotes the set of matrices $N$ such that 1) $N_{i j}<0$ (resp., $N_{i j}>0$ ), only if $M_{i j}<0$ (resp., $\left.M_{i j}>0\right)$, and 2) each row contains at least one nonzero entry. Recall that $\mathcal{Q}(M)$ is defined in Subsection 2.2.

Proposition 4. Let $I=(V, D=\{0,1\}, \mathcal{C})$ be a SAT instance, where $\mathcal{C}$ is given by a $C N F$ $\varphi$. Then we have the following 3 statements.

(i) $\operatorname{cl}\left(K_{\gg}(M)\right) \subseteq Y\left(P_{\text {presv }}(I)\right)$ for all $M \in \mathcal{Q}_{0}\left(M_{\varphi}\right)$.

(ii) $K_{\geq}(M) \subseteq Y\left(P_{\text {autark }}(I)\right)$ for all $M \in \mathcal{Q}\left(M_{\varphi}\right)$.

(iii) $K_{\gg}(M) \subseteq Y\left(P_{\text {sat }}(I)\right)$ for all $M \in \mathcal{Q}_{0}\left(M_{\varphi}\right)$.

Proof. Since (ii) is known by Kullmann (2003, Lemma 3.5), we show (i) and (iii) only. We first show (iii).

(iii): Let $y$ be a vector in $K_{\gg}(M)$. Then for each $c \in \varphi$, there exists an element $j$ such that $\left(x_{j} \in c\right.$ and $\left.y_{j}>0\right)$ or $\left(\bar{x}_{j} \in c\right.$ and $\left.y_{j}<0\right)$ hold. In either case, $a^{y}$ should satisfy $c$, implying that it satisfies $\varphi$.

(i): We have $K_{\gg}(M) \subseteq Y\left(P_{\text {sat }}(I)\right)$ for any $M \in \mathcal{Q}_{0}\left(M_{\varphi}\right)$ by (iii). By taking the closure of $K_{\gg}(M)$ and $Y\left(P_{\text {sat }}(I)\right)$, we obtain $\operatorname{cl}\left(K_{\gg}(M)\right) \subseteq \operatorname{cl}\left(Y\left(P_{\text {sat }}(I)\right)\right)$. Note that $\operatorname{cl}\left(Y\left(P_{\text {sat }}(I)\right)\right) \subseteq \operatorname{cl}\left(Y\left(P_{\text {presv }}\right)\right)=Y\left(P_{\text {presv }}(I)\right)$ holds, where the last equality follows from Proposition 1. Thus we have $\operatorname{cl}\left(K_{\gg}(M)\right) \subseteq Y\left(P_{\text {presv }}(I)\right)$.

We note that the proof of (iii) in Proposition 4 is similar to the one that $K_{\gg}(M) \subseteq$ $Y\left(P_{\text {sat }}(I)\right)$ for all $M \in \mathcal{Q}\left(M_{\varphi}\right)$ by Kullmann (2003, Lemma 3.2).

By Proposition 4 , a set $P$ of partial assignments is called weighted linear autark if we have $P=P\left(K_{\geq}(M)\right)$ for an $M \in \mathcal{Q}\left(M_{\varphi}\right)$, and weighted linear satisfying if we have $P=P\left(K_{\gg}(M)\right)$ for an $M \in \mathcal{Q}_{0}\left(M_{\varphi}\right)$.

The next example shows that weighted linear satisfiability preserving assignments dominate unweighted ones, which implies that weighed linear satisfiability preserving assignments are more useful than unweighted ones.

Example 8. Consider again the CSP instance $(V, D, \mathcal{C})$ given in Example 7 , i.e., $V=$ $\left\{x_{1}, x_{2}, x_{3}\right\}, D=\{0,1\}$ and $\mathcal{C}$ is given by a $C N F \varphi=\left(x_{1} \vee x_{2} \vee x_{3}\right)\left(x_{1} \vee \bar{x}_{2} \vee \bar{x}_{3}\right)\left(\bar{x}_{1} \vee x_{2} \vee\right.$ $\left.\bar{x}_{3}\right)\left(\bar{x}_{1} \vee \bar{x}_{2} \vee x_{3}\right)$. Recall that there exists no nontrivial unweighted linear autark assignment nor unweighted linear satisfying partial assignment for $\varphi$. On the other hand, define matrices $M_{1} \in \mathcal{Q}_{0}\left(M_{\varphi}\right)$ and $M_{2} \in \mathcal{Q}\left(M_{\varphi}\right)$ by

$$
M_{1}=\left(\begin{array}{lll}
1 & 1 & 1 \\
1 & 0 & 0 \\
0 & 1 & 0 \\
0 & 0 & 1
\end{array}\right) \text { and } M_{2}=\left(\begin{array}{rrr}
1 & 1 & 1 \\
2 & -1 & -1 \\
-1 & 2 & -1 \\
-1 & -1 & 2
\end{array}\right)
$$

Then we have

$$
\begin{gathered}
P\left(\operatorname{cl}\left(K_{\gg}\left(M_{1}\right)\right)\right)=\{(1,1,1),(1,1, *),(1, *, 1),(1, *, *),(*, 1,1), \\
(*, 1, *),(*, *, 1),(*, *, *)\}, \\
P\left(K_{\geq}\left(M_{2}\right)\right)=P\left(K_{\gg}\left(M_{1}\right)\right)=\{(1,1,1),(*, *, *)\} .
\end{gathered}
$$

These provide weighted linear satisfiability preserving assignments. For example, $(1,1, *)$ is $a \operatorname{cl}\left(K_{\gg}\left(M_{1}\right)\right)$-linear satisfiability preserving assignment. 
We note that we cannot replace $\operatorname{cl}\left(K_{\gg}(M)\right)$ with $K_{\geq}(M)$ in Proposition 4 (i). Namely, for a $\mathrm{CNF} \varphi$ and a matrix $M \in \mathcal{Q}_{0}\left(M_{\varphi}\right), K_{\geq}(M)$ is not always contained in $Y\left(P_{\text {presv }}(I)\right)$. For example, consider $\varphi=\left(x_{1} \vee x_{2}\right)\left(x_{1} \vee \bar{x}_{2}\right)$ and

$$
M=\left(\begin{array}{rr}
0 & 1 \\
0 & -1
\end{array}\right) \in \mathcal{Q}_{0}\left(M_{\varphi}\right) .
$$

$(-1,0)$ is clearly contained in $K_{\geq}(M)$, while not in $P_{\text {presv }}(I)$, since $(0, *)$ is not satisfiability preserving. Therefore we have $\bar{K}_{\geq}(M) \nsubseteq P_{\text {presv }}(I)$.

We also note that $\left\{\operatorname{cl}\left(K_{\gg}(M)\right): M \in \mathcal{Q}_{0}\left(M_{\varphi}\right)\right\}$ and $\left\{K_{\geq}(M): M \in \mathcal{Q}\left(M_{\varphi}\right)\right\}$ are incomparable in general. For example, for $\varphi=\left(x_{1} \vee x_{2}\right)$ and

$$
M=\left(\begin{array}{ll}
1 & 0
\end{array}\right) \in \mathcal{Q}_{0}\left(M_{\varphi}\right),
$$

there exists no matrix $M^{\prime} \in \mathcal{Q}\left(M_{\varphi}\right)$ such that $\operatorname{cl}\left(K_{\gg}(M)\right) \subseteq K_{\geq}\left(M^{\prime}\right)$. Moreover, for $\varphi=\left(x_{1} \vee \bar{x}_{2}\right)\left(\bar{x}_{1} \vee x_{2}\right)$ and

$$
M=\left(\begin{array}{rr}
1 & -1 \\
-1 & 1
\end{array}\right) \in \mathcal{Q}\left(M_{\varphi}\right)
$$

there exists no matrix $M^{\prime} \in \mathcal{Q}_{0}\left(M_{\varphi}\right)$ such that $K_{\geq}(M) \subseteq \operatorname{cl}\left(K_{\gg}\left(M^{\prime}\right)\right)$.

The following proposition shows that the linear autark and satisfying partial assignments in Proposition 4 are enough to find autark and satisfying partial assignments.

Proposition 5 (Kullmann, 2003, Lemmas 3.2 and 3.5). Let $I=(V, D, \mathcal{C})$ be a SAT instance, where $\mathcal{C}$ is given by a CNF $\varphi$.

(i) For any $A \in P_{\text {autark }}(I)$, there exists a matrix $M \in \mathcal{Q}\left(M_{\varphi}\right)$ such that $A$ is $K_{\geq}(M)$-linear.

(ii) For any $A \in P_{\text {sat }}(I)$, there exists a matrix $M \in \mathcal{Q}\left(M_{\varphi}\right)$ such that $A$ is $K_{\gg}(M)$-linear.

We next study the maximality of these linear satisfiability preserving assignments, which can be used to heuristically find a satisfiability preserving assignment.

Theorem 3. Let $I=(V, D, \mathcal{C})$ be a $S A T$ instance, where $\mathcal{C}$ is given by a $C N F \varphi$.

(i) Let $N$ be a matrix that represents linear autark assignments, i.e., $K_{\geq}(N)$ is contained in $Y\left(P_{\text {autark }}(I)\right)$. Then there exists a matrix $M$ in $\mathcal{Q}\left(M_{\varphi}\right)$ such that $\bar{K}_{\geq}(N) \subseteq K_{\geq}(M)$.

(ii) Let $N$ be a matrix that represents linear satisfying partial assignments, i.e., $K_{\gg}(N)$ is contained in $Y\left(P_{\text {sat }}(I)\right)$. Then there exists a matrix $M$ in $\mathcal{Q}_{0}\left(M_{\varphi}\right)$ such that $K_{\gg}(N) \subseteq$ $K_{\gg}(M)$.

Proof. (i): For a clause $c=\bigvee_{j \in J_{+}} x_{j} \vee \bigvee_{j \in J_{-}} \bar{x}_{j}$ in $\varphi$ with $J_{+} \cap J_{-}=\emptyset$, let $Z_{c}$ be a non-closed cone defined by $Z_{c}=\left\{y \in \mathbb{R}^{n}: y_{j} \leq 0\left(j \in J_{+}\right), y_{j} \geq 0\left(j \in J_{-}\right),-\sum_{j \in J_{+}} y_{j}+\sum_{j \in J_{-}} y_{j}>\right.$ $0\}$. Then $Z_{c}$ contains no point in $Y\left(P_{\text {autark }}(I)\right)$ and thus $K_{\geq}(N) \cap Z_{c}=\emptyset$ holds. Therefore, there exists a separating hyperplane between $K_{\geq}(N)$ and $Z_{c}$, i.e., there exists a vector $\alpha \in \mathbb{R}^{n}$ such that $K_{\geq}(N) \subseteq\left\{y \in \mathbb{R}^{n}: \alpha y \geq 0\right\}$ and $Z_{c} \subseteq\left\{y \in \mathbb{R}^{n}: \alpha y<0\right\}$ hold (e.g., Rockafellar, 1970, Thm. 11.7). We claim that $\alpha_{j}>0$ for all $j \in J_{+}, \alpha_{j}<0$ for all $j \in J_{-}$, and $\alpha_{j}=0$ for all $j \notin J_{+} \cup J_{-}$. 
Let $e_{j}$ denote the $j$ th unit vector. Since $-e_{j}$ and $e_{j}$ are respectively contained in $Z_{c}$ for $j \in J_{+}$and $j \in J_{-}$, we have $\alpha\left(-e_{j}\right)<0$ and $\alpha e_{j}<0$ respectively, which proves the claim for $j \in J_{+}$and $j \in J_{-}$. For $j \notin J_{+} \cup J_{-}$, define two vectors $z$ and $z^{\prime}$ by $z_{j}=1, z_{j}^{\prime}=-1$, $z_{k}=z_{k}^{\prime}=0$ if $k \notin J_{+} \cup J_{-} \cup\{j\}, z_{k}=z_{k}^{\prime}=-\varepsilon$ if $k \in J_{+}$, and $z_{k}=z_{k}^{\prime}=+\varepsilon$ if $k \in J_{-}$for an $\varepsilon>0$. Then $z$ and $z^{\prime}$ are contained in $Z_{c}$ and hence we have $\alpha z<0$ and $\alpha z^{\prime}<0$. This implies that $-\varepsilon\left(\sum_{k \in J_{+}} \alpha_{k}-\sum_{k \in J_{-}} \alpha_{k}\right)<\alpha_{j}<\varepsilon\left(\sum_{k \in J_{+}} \alpha_{k}-\sum_{k \in J_{-}} \alpha_{k}\right)$. Since $\varepsilon>0$ is arbitrary, we have $\alpha_{j}=0$.

Since $c$ is arbitrary, the claim implies (i) in the theorem.

(ii): For a clause $c=\bigvee_{j \in J_{+}} x_{j} \vee \bigvee_{j \in J_{-}} \bar{x}_{j}$ in $\varphi$ with $J_{+} \cap J_{-}=\emptyset$, let $Z_{c}=\{y \in$ $\left.\mathbb{R}^{n}: y_{j} \leq 0\left(j \in J_{+}\right), y_{j} \geq 0\left(j \in J_{-}\right)\right\}$. Then $Z_{c}$ is a closed cone which contains no point in $Y\left(P_{\text {sat }}(I)\right)$. This implies that $K_{\gg}(N) \cap Z_{c}=\emptyset$ holds. Since $K_{\gg}(N)$ is open, there exists a vector $\alpha \in \mathbb{R}^{n}$ such that $K_{\gg}(N) \subseteq\left\{y \in \mathbb{R}^{n}: \alpha y>0\right\}$ and $Z_{c} \subseteq\left\{y \in \mathbb{R}^{n}: \alpha y \leq 0\right\}$. We claim that $\alpha_{j} \geq 0$ for all $j \in J_{+}, \alpha_{j} \leq 0$ for all $j \in J_{-}$, and $\alpha_{j}=0$ for all $j \notin J_{+} \cup J_{-}$.

Since $-e_{j}, e_{j}$ and $\pm e_{j}$ are respectively contained in $Z_{c}$ for $j \in J_{+}, j \in J_{-}$and $j \notin J_{+} \cup J_{-}$, we have $\alpha\left(-e_{j}\right) \leq 0, \alpha e_{j} \leq 0$ and $\alpha\left( \pm e_{j}\right) \leq 0$ respectively. This proves the claim.

Since $\alpha \neq 0$ and $c$ is arbitrary, the claim implies (ii) in the theorem.

Note that (i) in Theorem 3 extends Kullmann's result (Proposition 5 (i)), since for any autark assignment, there exists a polyhedral cone containing a vector corresponding to the assignment.

By Theorem 3, $\left\{K_{\geq}(M): M \in \mathcal{Q}\left(M_{\varphi}\right)\right\}$ and $\left\{K_{\gg}(M): M \in \mathcal{Q}_{0}\left(M_{\varphi}\right)\right\}$ represent families of convex sets which are large in $Y\left(P_{\text {autark }}(I)\right)$ and $Y\left(P_{\text {sat }}(I)\right)$. However, the polyhedral cones respectively might not be maximal convex subsets of $Y\left(P_{\text {autark }}(I)\right)$ and $Y\left(P_{\text {sat }}(I)\right)$, as the next examples show. Here a convex subset $Z \subseteq Y(P)$ is called a maximal convex subset of $Y(P)$ if there exists no distinct convex set $Z^{\prime} \subseteq Y(P)$ that contains $Z$.

Example 9. Let $I=\left(V=\left\{x_{1}, x_{2}\right\}, D=\{0,1\}, \mathcal{C}\right)$ be a SAT instance, where $\mathcal{C}$ is given by a CNF $\varphi=x_{1}\left(x_{1} \vee x_{2}\right)$. For any $M \in \mathcal{Q}\left(M_{\varphi}\right), K_{\geq}(M)$ is not a maximal convex subset of $Y\left(P_{\text {autark }}(I)\right)$. Note that $Y\left(P_{\text {autark }}(I)\right)=\left\{y \in \mathbb{R}^{2}: y_{1}>0\right\} \cup\left\{y \in \mathbb{R}^{2}: y_{1} \geq\right.$ $\left.0, y_{2} \geq 0\right\}$ holds. For any $M \in \mathcal{Q}\left(M_{\varphi}\right)$, let $y=\left(M_{22},-M_{21}-1\right)$. Then $y$ is contained in $Y\left(P_{\text {autark }}(I)\right)$, but not in $K_{\geq}(M)$, since it violates $M_{21} y_{1}+M_{22} y_{2} \geq 0$. Moreover, we can see that $\operatorname{conv}\left(K_{\geq}(M) \cup\{y\}\right)$ is contained in $Y\left(P_{\text {autark }}(I)\right)$. Hence, there exists a convex set contained in $Y\left(P_{\text {autark }}(I)\right)$ that strictly contains $K_{\geq}(M)$, implying that $K_{\geq}(M)$ is not a maximal convex set in $Y\left(P_{\text {autark }}(I)\right)$.

We recall that, by Proposition 2 (i), for any matrix $N$ that represents linear satisfiability preserving assignments, there exists a matrix $M$ such that $K_{\geq}(N) \subseteq K_{\geq}(M) \subseteq Y\left(P_{\text {presv }}(I)\right)$. Example 9 shows that this $M$ does not belong to $\mathcal{Q}\left(M_{\varphi}\right)$ in general.

Example 10. Let $I=\left(V=\left\{x_{1}, x_{2}\right\}, D=\{0,1\}, \mathcal{C}\right)$ be a SAT instance, where $\mathcal{C}$ is given by a CNF $\varphi=x_{1} \vee x_{2}$. Then no $M \in \mathcal{Q}_{0}\left(M_{\varphi}\right)$ produces a maximal convex set $K_{\gg}(M)$ of $Y\left(P_{\text {sat }}(I)\right)$. This is because $\operatorname{conv}\left(K_{\gg}(M) \cup\{y\}\right) \subseteq Y\left(P_{\text {sat }}(I)\right)$ holds for $y=\left(M_{12},-M_{11}\right) \notin$ $K_{\gg}(M)$ if $M_{12} \neq 0$, and $y=(0,1) \notin K_{\gg}(M)$ if $M_{12}=0$.

Note that Example 10 does not contradict Proposition 2 (ii), since Example 10 deals with non-open convex sets. 
The next theorem shows that there exist maximal elements in $\left\{K_{\geq}(M): M \in \mathcal{Q}\left(M_{\varphi}\right)\right\}$ and $\left\{K_{\gg}(M): M \in \mathcal{Q}_{0}\left(M_{\varphi}\right)\right\}$ in terms of partial assignments, which contrasts with Examples 9 and 10 .

Theorem 4. Let $I=(V, D, \mathcal{C})$ be a $S A T$ instance, where $\mathcal{C}$ is given by a CNF $\varphi$.

(i) For any convex set $C$ contained in $Y\left(P_{\text {autark }}(I)\right)$, there exists a matrix $M \in \mathcal{Q}\left(M_{\varphi}\right)$ such that $P(C) \subseteq P\left(K_{\geq}(M)\right)$.

(ii) For any convex set $C$ contained in $Y\left(P_{\text {sat }}(I)\right)$, there exists a matrix $M \in \mathcal{Q}_{0}\left(M_{\varphi}\right)$ such that $P(C) \subseteq P\left(K_{\gg}(M)\right)$.

Proof. Since we have $3^{n}$ partial assignments, there exist finite points $y^{(1)}, \ldots, y^{(\ell)} \in C$ such that $P\left(\left\{y^{(1)}, \ldots, y^{(\ell)}\right\}\right)=P(C)$.

For (i), let $Z$ denote the convex cone closure of $y^{(i)}(i=1, \ldots, \ell)$. It follows that $Z \subseteq Y\left(P_{\text {autark }}(I)\right)$, since $C$ is convex and $Y\left(P_{\text {autark }}(I)\right)$ is a cone. By Theorem 3 , there exists a matrix $M \in \mathcal{Q}\left(M_{\varphi}\right)$ such that $Z \subseteq K_{\geq}(M)$ holds. Then we have $P(C) \subseteq P(Z) \subseteq$ $P\left(K_{\geq}(M)\right)$.

For (ii), let $Z$ denote the convex hull of $y^{(i)}(i=1, \ldots, \ell)$. It follows from convexity of $C$ that $Z \subseteq C \subseteq Y\left(P_{\text {sat }}(I)\right)$. By applying a similar argument in the proof of Theorem 3, we have $Z \subseteq K_{\gg}(M)$ for some $M \in \mathcal{Q}_{0}\left(M_{\varphi}\right)$.

We remark that $K_{\geq}\left(M_{\varphi}\right)$ or $K_{\gg}\left(M_{\varphi}\right)$ might not be maximal as seen in Examples 7 and 8.

\subsection{Maximality of Weighted Linear Satisfiability Preserving Assignments for Monotone CNFs}

In this subsection we investigate convex inner approximation of satisfiability preserving assignments for monotone and prime monotone CNFs. We show that several interesting properties hold for these CNFs, which are not satisfied for general CNFs.

Recall that a $\mathrm{CNF} \varphi$ is called monotone if it contains no negative literal.

Theorem 5. Let $I=(V, D, \mathcal{C})$ be a monotone $S A T$ instance, i.e., a $S A T$ instance where $\mathcal{C}$ is given by a monotone $C N F \varphi$. Then for any matrix $M \in \mathcal{Q}\left(M_{\varphi}\right)$, we have $P\left(K_{\geq}(M)\right)=$ $P_{\text {autark }}(I)$ and $P\left(K_{\gg}(M)\right)=P_{\text {sat }}(I)$.

Proof. We first show the case of $P_{\text {sat }}(I)$. For a satisfying partial assignment $A$, let $y$ be a vector in $\mathbb{R}^{n}$ defined as $y_{j}=\gamma$ if $a_{j}=1, y_{j}=-1$ if $a_{j}=0$, and $y_{j}=0$ otherwise (i.e., $\left.a_{j}=*\right)$, where $\gamma$ is a sufficiently large real. Then it is clear that partial assignment $a^{y}$ is equal to $A$. Moreover, we have $M y \gg 0$.

We can similarly show the case of autark assignments.

Theorem 5 does not hold if we replace $\mathcal{Q}\left(M_{\varphi}\right)$ by $\mathcal{Q}_{0}\left(M_{\varphi}\right)$ for satisfying partial assignments. For example, for a monotone SAT instance $I$ given by a monotone CNF $\varphi=x_{1} \vee x_{2}$, let $M=\left(\begin{array}{ll}1 & 0\end{array}\right) \in \mathcal{Q}_{0}\left(M_{\varphi}\right)$. Then we have $P\left(K_{\gg}(M)\right)=\{(1,0),(1,1),(1, *)\} \subsetneq P_{\text {sat }}(I)$.

We also remark that by Examples 9 and 10, even if $\varphi$ is a monotone CNF, there does not always exist an $M \in \mathcal{Q}\left(M_{\varphi}\right)$ such that $K_{\geq}(M)$ (resp., $K_{\gg}(M)$ ) is a maximal convex subset of $Y\left(P_{\text {autark }}(I)\right)$ (resp., $\left.Y\left(P_{\text {sat }}(I)\right)\right)$. This contrasts with Theorem 5 . 
We next consider prime monotone CNFs. Recall that a $\mathrm{CNF} \varphi$ is prime if replacing a clause $c \in \varphi$ by a proper subclause of $c$ produces a CNF which is not equivalent to $\varphi$. In this case, for autark assignments, maximality holds in terms of not only partial assignments but also convex sets in $Y\left(P_{\text {autark }}(I)\right)$.

Theorem 6. Let $I=(V, D, \mathcal{C})$ be a prime monotone $S A T$ instance, i.e., a SAT instance, where $\mathcal{C}$ is given by a prime monotone $C N F \varphi$. Then any $M \in \mathcal{Q}\left(M_{\varphi}\right)$ defines a maximal convex subset $K_{\geq}(M)$ of $Y\left(P_{\text {autark }}(I)\right)$.

Proof. We claim that $\operatorname{conv}\left(K_{\geq}(M) \cup\{y\}\right) \nsubseteq Y\left(P_{\text {autark }}(I)\right)$ holds for any $y \notin K_{\geq}(M)$. Fix a vector $y \notin K_{\geq}(M)$. Then $\sum_{x_{j} \in c_{i}} M_{i j} y_{j}+\varepsilon=0$ holds for some $c_{i} \in \varphi$ and $\varepsilon>0$. If $\left|c_{i}\right|=1$, then $M_{i j} y_{j}<0$ holds for the only $x_{j} \in c_{i}$, implying that $y_{j}<0$. Hence, $a^{y}$ does not satisfy $c_{i}$ and $y$ is not contained in $Y\left(P_{\text {autark }}(I)\right)$, which proves the claim. We therefore assume that $\left|c_{i}\right| \geq 2$.

Let $z$ be a vector in $\mathbb{R}^{n}$ defined as $z_{j}=\frac{1}{M_{i j}}\left(\sum_{x_{k} \in c_{i}, k \neq j} M_{i k} y_{k}+\frac{\left|c_{i}\right|-1}{\left|c_{i}\right|} \varepsilon\right)$ if $x_{j} \in c_{i}$, and $z_{j}=\gamma$ otherwise, where $\gamma$ is sufficiently large. We show that $z \in K_{\geq}(M)$ and $(y+$ $z) / 2 \notin Y\left(P_{\text {autark }}(I)\right)$ hold, which prove the claim since the former condition implies that $(y+z) / 2 \in \operatorname{conv}\left(K_{\geq}(M) \cup\{y\}\right)$ holds.

In order to prove that $z \in K_{\geq}(M)$ holds, we show that $\sum_{x_{j} \in c_{i^{\prime}}} M_{i^{\prime} j} z_{j} \geq 0$ holds for each $c_{i^{\prime}} \in \varphi$. If $c_{i^{\prime}}=c_{i}$, we have

$$
\begin{aligned}
\sum_{x_{j} \in c_{i}} M_{i j} z_{j} & =\sum_{x_{j} \in c_{i}}\left(\sum_{x_{k} \in c_{i}, k \neq j} M_{i k} y_{k}+\frac{\left|c_{i}\right|-1}{\left|c_{i}\right|} \varepsilon\right) \\
& =\sum_{x_{j} \in c_{i}} \sum_{x_{k} \in c_{i}} M_{i k} y_{k}-\sum_{x_{j} \in c_{i}} M_{i j} y_{j}+\left(\left|c_{i}\right|-1\right) \varepsilon \\
& =\left(\left|c_{i}\right|-1\right)\left(\sum_{x_{j} \in c_{i}} M_{i j} y_{j}+\varepsilon\right) \\
& =0 .
\end{aligned}
$$

On the other hand, if $c_{i^{\prime}} \neq c_{i}$, we have $\sum_{x_{j} \in c_{i^{\prime}}} M_{i^{\prime} j} z_{j} \geq 0$, since $c_{i^{\prime}}$ contains at least one variable not contained in $V\left(c_{i}\right)$ by primality of $\varphi$ and $\gamma$ is sufficiently large.

Next, we show that $(y+z) / 2 \notin Y\left(P_{\text {autark }}(I)\right)$ holds. For each $x_{j} \in c_{i}$,

$$
\begin{aligned}
y_{j}+z_{j} & =y_{j}+\frac{1}{M_{i j}}\left(\sum_{x_{k} \in c_{i}, k \neq j} M_{i k} y_{k}+\frac{\left|c_{i}\right|-1}{\left|c_{i}\right|} \varepsilon\right) \\
& =\frac{1}{M_{i j}}\left(\sum_{x_{j} \in c_{i}} M_{i j} y_{j}+\frac{\left|c_{i}\right|-1}{\left|c_{i}\right|} \varepsilon\right) \\
& =\frac{1}{M_{i j}}\left(-\varepsilon+\frac{\left|c_{i}\right|-1}{\left|c_{i}\right|} \varepsilon\right) \\
& <0 .
\end{aligned}
$$

Hence, a partial assignment $a^{(y+z) / 2}$ does not satisfy $c_{i}$, implying that $(y+z) / 2$ is not contained in $Y\left(P_{\text {autark }}(I)\right)$. This completes the proof.

Different from Theorem $6, K_{\gg}(M)$ for $M \in \mathcal{Q}_{0}\left(M_{\varphi}\right)$ is not always a maximal convex subset of $P_{\text {sat }}(I)$, which follows from Example 10 .

We also note that primality alone does not always yield maximality.

Example 11. Let $I=\left(V=\left\{x_{1}, x_{2}\right\}, D=\{0,1\}, \mathcal{C}\right)$ be a SAT instance, where $\mathcal{C}$ is given by a prime $C N F \varphi=\left(x_{1} \vee \bar{x}_{2}\right)\left(\bar{x}_{1} \vee x_{2}\right)$. Then no $M \in \mathcal{Q}\left(M_{\varphi}\right) \backslash\left\{M_{\varphi}\right\}$ produces a maximal convex subset $K_{\geq}(M)$ of $Y\left(P_{\text {autark }}(I)\right)$. 
Indeed, let $Z_{+}=\left\{y \in \mathbb{R}^{2}: y_{1}>0, y_{2}>0\right\}$ and $Z_{-}=\left\{y \in \mathbb{R}^{2}: y_{1}<0, y_{2}<0\right\}$. Then we have $Y\left(P_{\text {autark }}(I)\right)=Z_{+} \cup Z_{-} \cup\{0\}$. For any $M \in \mathcal{Q}\left(M_{\varphi}\right) \backslash\left\{M_{\varphi}\right\}$, it holds that $K_{\geq}(M) \subseteq Z_{+} \cup\{0\}$ or $K_{\geq}(M) \subseteq Z_{-} \cup\{0\}$. If $K_{\geq}(M) \subseteq Z_{+} \cup\{0\}$, then there exists a $y \in Z_{+}$which is not contained in $K_{\geq}(M)$, since $K_{\geq}(M)$ is closed and $Z_{+}$is open. It follows from convexity of $Z_{+} \cup\{0\}$ that $\operatorname{conv}\left(K_{\geq}(M) \cup\{y\}\right) \subseteq Z_{+} \cup\{0\}$. Hence, $K_{\geq}(M)$ is not a maximal convex subset of $Y\left(P_{\text {autark }}(I)\right)$. The case of $K_{\geq}(M) \subseteq Z_{-} \cup\{0\}$ can be treated in a similar way.

We remark that maximality in Theorem 6 is used to heuristically compute a locally fixable assignment, which can be found in the next section.

\section{Computing a Satisfiability Preserving Assignment for CSPs Given by Integer Linear Systems}

In this section, we discuss how to compute a linear satisfiability preserving assignment of CSPs given by integer linear systems. It is known that computing a nontrivial satisfiability preserving assignment defined in Section 2 is NP-hard, since we can solve the CSP by iteratively computing such partial assignments. We also note that it is still NP-hard to compute a partial assignment which is contained in some linear satisfiability preserving assignments, by Proposition 5. Hence, we consider restricting linear satisfiability preserving assignments.

For CNFs $\varphi$, it is natural to consider linear autark assignments derived from matrices in $\mathcal{Q}\left(M_{\varphi}\right)$, in particular, unweighted linear autark assignments. For the 0-1 integer linear systems, we note that each constraint is unate (i.e., it can be represented by a monotone CNF by changing the polarity of variables appropriately). By Theorems 5 and 6 , unweighted linear autark (i.e., globally fixable) assignments for its prime CNF expression are maximal in terms of both the set of the corresponding vectors and partial assignments. We thus try to use the prime CNF expression of each inequality to obtain a maximal linear globally fixable assignment of each inequality. However, we note that a prime CNF of an inequality might have exponentially many clauses with respect to the number of variables in the inequality. This implies that we cannot explicitly have linear inequalities that represent unweighted linear globally fixable assignments.

In this section, we study computing a linear locally fixable assignment which is contained in the intersection of such unweighted linear globally fixable assignments. We show that it can be computed in pseudo-polynomial time, even if some constraints have a non-fixed number of variables. As a corollary, we show that TVPI, Horn, and q-Horn systems are solvable in pseudo-polynomial time by repeatedly computing such linear partial assignments, which is discussed in Subsection 4.2. We assume that $d=|D|$ is a part of the input throughout the section.

We first consider the Boolean case, i.e., 0-1 integer linear systems, and then turn to the general case.

\subsection{The Case of 0-1 Integer Linear Systems}

In this subsection, we discuss the complexity of computing a satisfiability preserving assignment of the Boolean CSP given by 0-1 integer linear systems. 
We consider a Boolean CSP instance $I=(V, D=\{0,1\}, \mathcal{C})$, where $\mathcal{C}$ is given by a linear system

$$
G x \geq h,
$$

where $G$ is a matrix in $\mathbb{Z}^{m \times n}$ and $h$ is a vector in $\mathbb{Z}^{m}$.

We note that each linear inequality in the system $(22)$ is unate. To see this, consider a vector $\alpha \geq 0$ and a real $\beta$. Then a linear inequality $\alpha x \geq \beta$ is monotone since $\alpha x \geq \beta$ and $x \leq x^{\prime}$ clearly imply $\alpha x^{\prime} \geq \beta$. For a general $\alpha$, by changing the polarity of the variables with $\alpha_{j}<0$, we obtain a monotone linear inequality, implying that $\alpha x \geq \beta$ is unate. It is known that every unate Boolean function has the unique prime CNF expression (e.g., Crama \& Hammer, 2011). Let $I_{i}$ denote the CSP instance that has a single constraint given as the $i$ th inequality of $(22)$. By Theorem $6, Y\left(P_{\text {g-fix }}\left(I_{i}\right)\right)$ is maximally (inner) approximated by $K_{\geq}\left(M_{\varphi_{i}}\right)$ for the unique prime CNF expression $\varphi_{i}$ of $I_{i}$, where primality implies $P_{\text {autark }}\left(I_{i}\right)=$ $P_{\text {g-fix }}\left(I_{i}\right)$ by Proposition 2 .

Consider a single inequality:

$$
\sum_{j \in J_{+}} g_{j} x_{j}-\sum_{j \in J_{-}} g_{j} x_{j} \geq \eta
$$

where $J_{+}, J_{-} \subseteq V, J_{+} \cap J_{-}=\emptyset, g_{j}>0$ for $j \in J_{+} \cup J_{-}$, and $\eta \in \mathbb{Z}$.

Let $U$ denote the set of the extreme infeasible points of (23), i.e., $u \in U$ satisfies that $u+e_{j}$ is feasible to (23) if $j \in J_{+}$and $u_{j}=0$, and $u-e_{j}$ is feasible to (23) if $j \in J_{-}$and $u_{j}=1$. Then it is known by, e.g., the work of Crama and Hammer (2011, Thm. 1.27) that $\varphi=\bigwedge_{u \in U}\left(\bigvee_{j \in J_{+}: u_{j}=0} x_{j} \vee \bigvee_{j \in J_{-}: u_{j}=1} \bar{x}_{j}\right)$ is the unique prime CNF representing (23), which implies the following lemma.

Lemma 1. Let $\varphi$ denote the unique prime CNF representing (23). Then we have

$$
K_{\geq}\left(M_{\varphi}\right)=\left\{y \in \mathbb{R}^{n} \mid \sum_{j \in J_{+}: u_{j}=0} y_{j}-\sum_{j \in J_{-}: u_{j}=1} y_{j} \geq 0(u \in U)\right\} .
$$

Let $\varphi_{i}$ denote the unique prime CNF expression for the $i$ th inequality in (22) for each $i$. Then we note that $\bigcap_{i=1}^{m} K_{\geq}\left(M_{\varphi_{i}}\right)$ represents linear locally fixable assignments. We show the following result for such assignments.

Theorem 7. We can find in pseudo-polynomial time a nontrivial linear locally fixable assignment in $\bigcap_{i=1}^{m} K_{\geq}\left(M_{\varphi_{i}}\right)$ for the 0-1 integer linear system (22), if it exists. Moreover, it is possible in polynomial time, for the unit integer linear system, i.e., the input matrix $G$ is given by $G \in\{0,-1,+1\}^{m \times n}$.

In order to find a nontrivial partial assignment in $\bigcap_{i=1}^{m} K_{\geq}\left(M_{\varphi_{i}}\right)$, we solve the following two linear programming (LP) problems $\mathrm{P}_{\ell}^{+}$and $\mathrm{P}_{\ell}^{-}$for each $x_{\ell} \in V$ :

$$
\begin{array}{rllll}
\text { Problem } \mathrm{P}_{\ell}^{+} & & \begin{array}{r}
\text { Problem } \mathrm{P}_{\ell}^{-} \\
\text {maximize }
\end{array} & -y_{\ell} \\
\text { maximize } & y_{\ell} & \text { subject to } & M_{\varphi_{i}} y \geq 0 \quad(i=1, \ldots, m) \\
\text { subject to } & M_{\varphi_{i}} y \geq 0 \quad(i=1, \ldots, m) & & -1 \leq y_{j} \leq 1(j=1, \ldots, n) .
\end{array}
$$


The constraints $-1 \leq y_{j} \leq 1$ for $j=1, \ldots, n$ in (25) ensure that the solution space is bounded. We note that the number of the inequalities in the LP problems (25) might be exponential in the size of the input. For example, for a linear inequality $\sum_{j=1}^{n} x_{j} \geq\lfloor n / 2\rfloor$, the set $U$ of the extreme infeasible points is given by

$$
U=\left\{u \in\{0,1\}^{n} \mid \sum_{j=1}^{n} u_{j}=\lfloor n / 2\rfloor-1\right\} .
$$

Hence $|U|=\left(\begin{array}{c}n \\ \lfloor n / 2\rfloor-1\end{array}\right)=\Omega\left(2^{n / 2-\log n}\right)$ holds, which implies that the LP problems (25) have exponentially many inequalities. Instead of solving (25) directly, we make use of the ellipsoid method described in Subsection 2.3. By Theorem 1 in Subsection 2.3, optimization is polynomially equivalent to solving the separation problem. Namely, given a $y \in \mathbb{R}^{n}$, we compute an inequality in (25) that is violated by $y$, if $y$ is infeasible. Since $-1 \leq y_{j} \leq 1$ can be checked efficiently, we consider how to check if $M_{\varphi_{i}} y \geq 0$ is satisfied for each $i$.

Let $J_{+}^{(i)}=\left\{j \in V: G_{i j}>0\right\}, J_{-}^{(i)}=\left\{j \in V: G_{i j}<0\right\}$ and $U^{(i)}$ denote the set of extreme infeasible points of the $i$ th constraint in (22). Then by Lemma 1, the following integer LP problem computes $\min _{k}\left\{\left(M_{\varphi_{i}}\right)_{k} y\right\}$, where $\left(M_{\varphi_{i}}\right)_{k}$ denotes the $k$ th row vector of $M_{\varphi_{i}}$.

$$
\begin{array}{ll}
\operatorname{minimize} & \sum_{j \in J_{+}^{(i)}} y_{j}\left(1-u_{j}\right)-\sum_{j \in J_{-}^{(i)}} y_{j} u_{j} \\
& \left(=-\sum_{j \in J_{+}^{(i)} \cup J_{-}^{(i)}} y_{j} u_{j}+\sum_{j \in J_{+}^{(i)}} y_{j}\right) \\
\text { subject to } & u \in U^{(i)} .
\end{array}
$$

Hence the optimal value of (27) is at least zero if and only if $M_{\varphi_{i}} y \geq 0$ holds. It is easy to see that $u \in U^{(i)}$ if and only if

1) $\sum_{j \in J_{+}^{(i)} \cup J_{-}^{(i)}} G_{i j} u_{j} \leq h_{i}-1$,

2) $u \in\{0,1\}^{n}$

3) $u$ is extreme, i.e.,

$$
\begin{cases}\sum_{j \in J_{+}^{(i)} \cup J_{-}^{(i)}} G_{i j} u_{j}+G_{i k} \geq h_{i} & \left(k \in J_{+}^{(i)}, u_{k}=0\right), \\ \sum_{j \in J_{+}^{(i)} \cup J_{-}^{(i)}} G_{i j} u_{j}-G_{i k} \geq h_{i} & \left(k \in J_{-}^{(i)}, u_{k}=1\right) .\end{cases}
$$

Although the condition 3) seems to be difficult to deal with, we show that the following dynamic programming approach can solve (27) in pseudo-polynomial time.

We assume that $0<\left|G_{i 1}\right| \leq \cdots \leq\left|G_{i n}\right|$ holds without loss of generality. For a vector $u \in\{0,1\}^{n}$, we say that $u$ chooses an element $j$ if either $\left(u_{j}=1\right.$ and $\left.j \in J_{+}^{(i)}\right)$ or $\left(u_{j}=0\right.$ and $j \in J_{-}^{(i)}$ ) holds. Let $k$ be the first element that is not chosen by $u$. Then we can see that $u$ is an extreme infeasible point of the $i$ th constraint if and only if $h_{i}-G_{i k}+1 \leq \sum_{j=1}^{n} G_{i j} u_{j} \leq h_{i}$ holds when $k \in J_{+}^{(i)}$, and $h_{i}+G_{i k}+1 \leq \sum_{j=1}^{n} G_{i j} u_{j} \leq h_{i}$ holds when $k \in J_{-}^{(i)}$. Therefore, in order to solve (27), it is enough to solve the following integer LP problem for each 
$k=1, \ldots, n+1$.

$$
\begin{array}{ll}
\operatorname{maximize} & \sum_{j=1}^{n} y_{j} u_{j} \\
\text { subject to } & h_{i}-G_{i k}+1 \leq \sum_{j=1}^{n} G_{i j} u_{j} \leq h_{i} \\
& \left(\text { resp. }, h_{i}+G_{i k}+1 \leq \sum_{j=1}^{n} G_{i j} u_{j} \leq h_{i}\right) \\
& k \text { is the first unchosen element by } u \\
& u \in\{0,1\}^{n} .
\end{array}
$$

Here $k=n+1$ means that $u$ chooses all the elements.

Let $k \in\{1, \ldots, n\}$. For an integer $\ell$ with $k \leq \ell \leq n$ and an integer $w \in \mathbb{Z}$, let $v(\ell, w)$ denote the objective value of

$$
\begin{array}{ll}
\operatorname{maximize} & \sum_{j=1}^{\ell} y_{j} u_{j} \\
\text { subject to } & \sum_{j=1}^{\ell} G_{i j} u_{j}=w \\
& k \text { is the first unchosen element by } u \\
& u \in\{0,1\}^{\ell} .
\end{array}
$$

Let us consider the case in which $k \in J_{+}^{(i)}$, since the case of $k \in J_{-}^{(i)}$ can be treated similarly. Then we have

$$
\begin{aligned}
& v(k, w)= \begin{cases}\sum_{j \in J_{+}^{(i)}: j \leq k-1} y_{j} & \left(w=\sum_{j \in J_{+}^{(i)}: j \leq k-1} G_{i j}\right), \\
-\infty & \text { (otherwise) }\end{cases} \\
& v(\ell, w)=\max \left\{v(\ell-1, w), v\left(\ell-1, w-G_{i \ell}\right)+y_{\ell}\right\} \quad(\ell=k+1, \ldots, n) .
\end{aligned}
$$

Hence, we can see that $\max _{h_{i}-G_{i k}+1 \leq w \leq h_{i}} v(n, w)$ is the optimal value of (28). Since the dynamic programming algorithm requires $\mathrm{O}\left((n-k) n \eta_{i}\right)$ time for each $k$, where $\eta_{i}=\max \left\{h_{i}\right.$, $\left.\left|G_{i 1}\right|, \ldots,\left|G_{i n}\right|\right\}$, we can solve the separation problem in $\mathrm{O}\left(n^{3} m \eta\right)$ time, where $\eta=\max _{i} \eta_{i}$. Hence, by Theorem 1, we can find a nontrivial linear locally fixable assignment for the 0-1 integer linear system (22) in pseudo-polynomial time.

We next consider the unit integer linear system, i.e., the integer linear system such that the input matrix $G$ is given by $G \in\{0,-1,+1\}^{m \times n}$. Note that this includes global cardinality constraints (van Hoeve \& Katriel, 2006) and is related to MAX(MIN) ONES (Creignou et al., 2001).

Let

$$
\sum_{j \in J_{+}} x_{j}+\sum_{j \in J_{-}}\left(1-x_{j}\right) \geq k
$$

be an inequality in the system. Then it has the unique prime CNF representing $\varphi$ defined by

$$
\varphi=\bigwedge_{\substack{I_{+} \subseteq J_{+}, I_{-} \subseteq J_{-}: \\\left|I_{+}\right|+\left|I_{-}\right|=\left|J_{+}\right|+\left|J_{-}\right|-k+1}}\left(\bigvee_{j \in I_{+}} x_{j} \vee \bigvee_{j \in I_{-}} \bar{x}_{j}\right)
$$

Thus, we have the following lemma.

Lemma 2. Let $\varphi$ be the unique prime CNF representing (31). Then we have

$$
\begin{aligned}
K_{\geq}\left(M_{\varphi}\right)=\{ & \left\{y \in \mathbb{R}^{n}: \sum_{j \in I_{+}} y_{j}-\sum_{j \in I_{-}} y_{j} \geq 0\right. \\
& \left.I_{+} \subseteq J_{+}, I_{-} \subseteq J_{-},\left|I_{+}\right|+\left|I_{-}\right|=\left|J_{+}\right|+\left|J_{-}\right|-k+1\right\} .
\end{aligned}
$$


Again the number of the constraints in the LP problems (25) might be exponential in the size of the input. Hence we again use the ellipsoid method to solve the LP problems $(25)$.

Lemma 3. We can solve the separation problem for the LP problems (25) in polynomial time if $G \in\{0,-1,+1\}^{m \times n}$.

Proof. Suppose that a vector $y \in \mathbb{R}^{n}$ is given. Then, we note that $y \in K_{\geq}\left(M_{\varphi_{1}}\right) \cap \cdots \cap$ $K_{\geq}\left(M_{\varphi_{m}}\right)$ holds if and only if

$$
\begin{aligned}
\min \left\{\sum_{j \in I_{+}} y_{j}-\sum_{j \in I_{-}} y_{j} \geq 0: I_{+} \subseteq J_{+}^{(i)}, I_{-} \subseteq J_{-}^{(i)}\right. \\
\left.\left|I_{+}\right|+\left|I_{-}\right|=\left|J_{+}^{(i)}\right|-h_{i}+1\right\} \geq 0
\end{aligned}
$$

holds for all $i=1, \ldots, m$. The left hand side of (34) can be computed simply by sorting the set $\left\{y_{j}: j \in J_{+}^{(i)}\right\} \cup\left\{-y_{j}: j \in J_{-}^{(i)}\right\}$ and summing the smallest $\left|J_{+}^{(i)}\right|-h_{i}+1$ elements. Hence, we can solve the separation problem in $\mathrm{O}(m n \log n)$ time.

We therefore proved Theorem 7 .

\subsection{The Case of General Integer Linear Systems}

In this subsection, we consider linear satisfiability preserving assignments for the nonBoolean CSP given by integer linear systems.

Let

$$
G x \geq h, x \in\{0,1, \ldots, d\}^{n}
$$

denote an integer linear system such that the $i$ th inequality is represented by

$$
\sum_{j \in J_{+}^{(i)}} \alpha_{i j} x_{j}-\sum_{j \in J_{-}^{(i)}} \alpha_{i j} x_{j} \geq h_{i}
$$

for $\alpha_{i j}>0\left(j \in J_{+}^{(i)} \cup J_{-}^{(i)}\right)$. Let $U_{i}$ denote the set of the extreme infeasible points of (36), i.e., $u \in U_{i}$ satisfies that $u+e_{j}$ is feasible to (36) if $j \in J_{+}$and $u_{j} \neq d$, and $u-e_{j}$ is feasible to (36) if $j \in J_{-}$and $u_{j} \neq 0$.

Recall the encoding that transform CSP instances with $D=\{0,1, \ldots, d\}$ to Boolean CSP instances in Section 1. Define a $\operatorname{CNF} \varphi_{i}$ by

$$
\varphi_{i}=\bigwedge_{u \in U_{i}}\left(\bigvee_{j \in J_{+}^{(i)}: u_{j} \neq d} x_{j, u_{j}+1} \vee \bigvee_{j \in J_{-}^{(i)}: u_{j} \neq 0} \bar{x}_{j, u_{j}}\right) .
$$

We note that $G x \geq h$ and $x \in D^{n}$ if and only if all $\varphi_{i}(i=1, \ldots, m)$ and $x_{j, p} \geq x_{j, p+1}(j=$ $1, \ldots, n ; p=1, \ldots, d-1)$ are satisfied. Since $x_{j, p} \geq x_{j, p+1}$ can be represented by a CNF

$$
\psi_{j, p}=\left(x_{j, p} \vee \bar{x}_{j, p+1}\right),
$$

by applying the ellipsoid method proposed in the previous subsection, we have the following result. 
Theorem 8. We can find in pseudo-polynomial time a nontrivial linear locally fixable assignment in $\bigcap_{i=1}^{m} K_{\geq}\left(M_{\varphi_{i}}\right) \cap \bigcap_{j, p} K_{\geq}\left(M_{\psi_{j, p}}\right)$ for the general integer linear system (22), if it exists.

We then show that TVPI, Horn, and q-Horn systems are solvable in pseudo-polynomial time by repeatedly computing linear partial assignments in Theorem 8. A system $G x \geq h$ is said to be TVPI if each row of $G$ contains at most two nonzero elements, Horn if each row of $G$ contains at most one positive element, and $q$-Horn if the optimal value of the following LP problem is at most one (Kimura \& Makino, 2016).

$$
\begin{array}{cll}
\operatorname{minimize} & \beta \\
\text { subject to } & \sum_{j: G_{i j}>0} \alpha_{j}+\sum_{j: G_{i j}<0}\left(1-\alpha_{j}\right) \leq \beta \quad(i=1, \ldots, m) \\
& 0 \leq \alpha_{j} \leq 1 & (j=1, \ldots, n) .
\end{array}
$$

Also, a CNF $\varphi=\bigwedge_{i=1}^{m}\left(\bigvee_{j \in J_{+}^{(i)}} x_{j} \vee \bigvee_{j \in J_{-}^{(i)}} \bar{x}_{j}\right)$ is called 2-CNF if $\left|J_{+}^{(i)} \cup J_{-}^{(i)}\right| \leq 2$ holds for all $i=1, \ldots, m$, Horn if $\left|J_{+}^{(i)}\right| \leq 1$ holds for all $i=1, \ldots, m$, and $q$-Horn if the optimal value of the following LP problem is at most one (Boros et al., 1990).

$$
\begin{array}{cll}
\operatorname{minimize} & \beta \\
\text { subject to } & \sum_{j \in J_{+}^{(i)}} \alpha_{j}+\sum_{j \in J_{-}^{(i)}}\left(1-\alpha_{j}\right) \leq \beta & (i=1, \ldots, m) \\
& 0 \leq \alpha_{j} \leq 1 & (j=1, \ldots, n) .
\end{array}
$$

We note that $\bigwedge_{i=1}^{m} \varphi_{i} \wedge \bigwedge_{j=1, \ldots, n ; p=1, \ldots, d-1} \psi_{j, p}$ is Horn (resp., TVPI (i.e., a 2-CNF) and q-Horn) if so is the original integer linear system $G x \geq h$ (Petke \& Jeavons, 2011). It is known by Kullmann (2000) and van Maaren (2000) that 2-, Horn and q-Horn CNFs can be solved by repeatedly finding nontrivial unweighted linear autark assignments. Therefore, we have the following result.

Theorem 9. If an integer linear system is TVPI, Horn or q-Horn, then it can be solved in pseudo-polynomial time by repeatedly finding linear locally fixable assignments in $\bigcap_{i=1}^{m}$ $K_{\geq}\left(M_{\varphi_{i}}\right) \cap \bigcap_{j, p} K_{\geq}\left(M_{\psi_{j, p}}\right)$.

Finally, we note that the following naive encoding does not work, since TVPI (resp., Horn and q-Horn) systems does not always transformed to the 0-1 TVPI (resp., Horn and q-Horn) systems.

$$
\left\{\begin{array}{rlrl}
G\left(\sum_{p=1}^{d} x_{p}\right) & \geq h & \\
x_{j, p} & \geq x_{j, p+1} & (j=1, \ldots, n ; p=1, \ldots, d-1) \\
x_{p} & \in\{0,1\}^{n} \quad(p=1, \ldots, d),
\end{array}\right.
$$

where $x_{p}=\left(x_{1, p}, \ldots, x_{n, p}\right)$ for $p=1, \ldots, d$.

\section{Acknowledgments}

The authors are indebted to the reviewers for their valuable comments and suggestions, which have considerably improved the presentation of the results. The authors also thank 
Dr. Yasushi Kawase for fruitful discussion on Theorem 7. The first author is supported by JSPS KAKENHI Grant Number JP15H06286. The second author is supported by JSPS KAKENHI Grant Number JP24106002, JP25280004, JP26280001, and JST CREST Grant Number JPMJCR1402, Japan.

\section{References}

Bar-Yehuda, R., \& Rawitz, D. (2001). Efficient algorithms for integer programs with two variables per constraint. Algorithmica, 29(4), 595-609.

Bordeaux, L., Cadoli, M., \& Mancini, T. (2008). A unifying framework for structural properties of CSPs: Definitions, complexity, tractability. Journal of Artificial Intelligence Research, 32, 607-629.

Boros, E., Crama, Y., \& Hammer, P. L. (1990). Polynomial-time inference of all valid implications for Horn and related formulae. Annals of Mathematics and Artificial Intelligence, 1, 21-32.

Brualdi, R. A., \& Shader, B. L. (1995). Matrices of Sign-Solvable Linear Systems. Cambridge University Press, Cambridge.

Bulatov, A. A. (2006). A dichotomy theorem for constraint satisfaction problems on a 3-element set. Journal of the ACM, 53, 66-120.

Bulatov, A. A. (2017). A dichotomy theorem for nonuniform CSPs. In Proceedings of the 58th IEEE Annual Symposium on Foundations of Computer Science, pp. 319-330.

Chandru, V., \& Hooker, J. (1999). Optimization Methods for Logical Inference. WileyInterscience, New York, USA.

Cook, S. (1971). The complexity of theorem proving procedures. In Proceedings of the Third Annual ACM Symposium on Theory of Computing, pp. 151-158.

Crama, Y., Ekin, O., \& Hammer, P. L. (1997). Variable and term removal from Boolean formulae. Discrete Applied Mathematics, 75, 217-230.

Crama, Y., \& Hammer, P. L. (2011). Boolean Functions: Theory, Algorithms, and Applications. Cambridge University Press, New York, USA.

Creignou, N., Khanna, S., \& Sudan, M. (2001). Complexity classifications of Boolean constraint satisfaction problems. Society for Industrial and Applied Mathematics, Pennsylvania, USA.

Davis, M., Logemann, G., \& Loveland, D. (1962). A machine program for theorem-proving. Communications of the ACM, 5, 394-397.

Davis, M., \& Putnam, H. (1960). A computing procedure for quantification theory. Journal of the ACM, 7, 201-215.

Dechter, R. (2003). Constraint Processing. Morgan Kaufmann, California, USA.

Even, S., Itai, A., \& Shamir, A. (1976). On the complexity of timetable and multicommodity flow problems. SIAM Journal on Computing, 5(4), 691-703. 
Freuder, E. C. (1991). Eliminating interchangeable values in constraint satisfaction problems. In Proceedings of the 9th National Conference on Artificial Intelligence, pp. $227-233$.

Garey, M. R., \& Johnson, D. S. (1979). Computers and Intractability: A Guide to the Theory of NP-Completeness. W. H. Freeman and Company, USA.

Gaspers, S., \& Szeider, S. (2012). Backdoors to satisfaction. In Bodlaender, H. L., Downey, R. G., Fomin, F. V., \& Marx, D. (Eds.), The Multivariate Algorithmic Revolution and Beyond, pp. 287-317. Springer-Verlag, Heidelberg, Germany.

Glover, F. (1964). A bound escalation method for the solution of integer linear programs. Cahiers du Centre d'Etudes de Recherche Operationelle, 6(3), 131-168.

Grötschel, M., Lovász, L., \& Schrijver, A. (1993). Geometric Algorithms and Combinatorial Optimization Second Corrected Edition. Springer-Verlag, Heidelberg, Germany.

Henschen, L. J., \& Wos, L. A. (1974). Unit refutations and Horn sets. Journal of the ACM, 21, 590-605.

Hochbaum, D. S., Megiddo, N., Naor, J., \& Tamir, A. (1993). Tight bounds and 2approximation algorithms for integer programs with two variables per inequality. Mathematical Programming, 62, 69-83.

Hoffman, A. J., \& Kruskal, J. B. (1956). Integral boundary points of convex polyhedra. In Khun, H., \& Tucker, A. (Eds.), Linear Inequalities and Related Systems, pp. 223-246. Princeton University Press, London, UK.

Jeavons, P. G., Cohen, D. A., \& Cooper, M. C. (1994). A substitution operation for constraints. In 2nd Workshop on Principles and Practice of Constraint Programming, pp. 1-9.

Jonsson, P., \& Krokhin, A. (2004). Recognizing frozen variables in constraint satisfaction problems. Theoretical Computer Science, 329, 93-113.

Kimura, K., \& Makino, K. (2016). Trichotomy for integer linear systems based on their sign patterns. Discrete Applied Mathematics, 200, 67-78.

Kleine Büning, H., \& Kullmann, O. (2009). Minimal unsatisfiability and autarkies. In Biere, A., Heule, M., van Maaren, H., \& Walsh, T. (Eds.), Handbook of Satisfiability, pp. 339-401. IOS Press, Amsterdam.

Kullmann, O. (2000). Investigations on autark assignments. Discrete Applied Mathematics, 10\%, 99-137.

Kullmann, O. (2003). Lean clause-sets: Generalizations of minimally unsatisfiable clausesets. Discrete Applied Mathematics, 130, 209-249.

Kullmann, O. (2011). Constraint satisfaction problems in clausal form I: Autarkies and deficiency. Fundamenta Informaticae, 109, 27-81.

Lagarias, J. C. (1985). The computational complexity of simultaneous Diophantine approximation problems. SIAM Journal on Computing, 14 (1), 196-209.

Lenstra, Jr., H. W. (1983). Integer programming with a fixed number of variables. Mathematics of Operations Research, 8, 538-548. 
Lewis, H. R. (1978). Renaming a set of clauses as a Horn set. Journal of the ACM, 25, $134-135$.

Monasson, R., Zecchina, R., Kirkpatrick, S., Selman, B., \& Troyansky, L. (1999). Determining computational complexity from characteristic 'phase transitions'. Nature, 400, $133-137$.

Monien, B., \& Speckenmeyer, E. (1985). Solving satisfaiability in less than $2^{n}$ steps. Discrete Applied Mathematics, 10, 287-295.

Papadimitriou, C. H. (1981). On the complexity of integer programming. Journal of the $A C M, 28(4), 765-768$.

Petke, J., \& Jeavons, P. (2011). The order encoding: From tractable CSP to tractable SAT. In Proceedings of the 14th International Conference on Theory and Applications of Satisfiability Testing, pp. 371-372.

Prestwich, S. (2004). Full dynamic substitutability by SAT encoding. In Principles and Practice of Constraint Programming, pp. 512-526.

Rockafellar, R. T. (1970). Convex Analysis. Princeton University Press, New Jersey, USA.

Rossi, F., van Beek, P., \& Walsh, T. (Eds.). (2006). Handbook of Constraint Programming. Elsevier Science, New York, USA.

Schaefer, T. J. (1978). The complexity of satisfiability problems. In Proceedings of the 10th annual ACM Symposium on Theory of Computing, pp. 216-226.

Tamura, N., Taga, A., Kitagawa, S., \& Banbara, M. (2009). Compiling finite linear CSP into SAT. Constraints, 14, 254-272.

Tsang, E. (1993). Foundations of Constraint Satisfaction. Academic Pr, London and San Diego.

van Hoeve, W.-J., \& Katriel, I. (2006). Global constraints. In Rossi, F., van Beek, P., \& Walsh, T. (Eds.), Handbook of Constraint Programming, pp. 169-208. Elsevier, New York, USA.

van Maaren, H. (2000). A short note on some tractable cases of the satisfiability problem. Information and Computation, 158, 125-130.

van Maaren, H., \& Dang, C. (2002). Simplicial pivoting algorithms for a tractable class of integer programs. Journal of Combinatorial Optimization, 6(2), 133-142.

Williams, R., Gomes, C., \& Selman, B. (2003). Backdoors to typical case complexity. In Proceedings of the 8th Internatinal Joint Conference on Artificial Intelligence, pp. 1173-1178.

Zhuk, D. (2017). A proof of CSP dichotomy conjecture. In Proceedings of the 58th IEEE Annual Symposium on Foundations of Computer Science, pp. 331-342. 